\title{
Construction and Analysis of Binary Subdivision Schemes for Curves and Surfaces Originated from Chaikin Points
}

\author{
Rabia Hameed and Ghulam Mustafa \\ Department of Mathematics, The Islamia University of Bahawalpur, Bahawalpur, Pakistan \\ Correspondence should be addressed to Ghulam Mustafa; ghulam.mustafa@iub.edu.pk
}

Received 23 August 2016; Revised 24 October 2016; Accepted 30 October 2016

Academic Editor: Jacques Liandrat

Copyright @ 2016 R. Hameed and G. Mustafa. This is an open access article distributed under the Creative Commons Attribution License, which permits unrestricted use, distribution, and reproduction in any medium, provided the original work is properly cited.

\begin{abstract}
We present a new variant of Lane-Riesenfeld algorithm for curves and surfaces both. Our refining operator is the modification of Chaikin/Doo-Sabin subdivision operator, while each smoothing operator is the weighted average of the four/sixteen adjacent points. Our refining operator depends on two parameters (shape and smoothing parameters). So we get new families of univariate and bivariate approximating subdivision schemes with two parameters. The bivariate schemes are the nontensor product schemes for quadrilateral meshes. Moreover, we also present analysis of our families of schemes. Furthermore, our schemes give cubic polynomial reproduction for a specific value of the shape parameter. The nonuniform setting of our univariate and bivariate schemes gives better performance than that of the uniform schemes.
\end{abstract}

\section{Introduction and Related Work}

Subdivision is a process of generating curves/surfaces by iteratively refining a set of control points according to some specific refinement rules. The set of these specific rules are called the subdivision scheme. For stationary, linear, and uniform subdivision schemes, these refinement rules are same at each refinement level. So the set of new control points at next refinement level can be generated by computing the affine combination of the set of control points at previous refinement level. A general compact form of linear, uniform, and stationary binary $m$-variate $(m=1,2)$ subdivision scheme $S_{a}$ which maps a polygon $f^{k-1}=\left\{f_{\alpha}^{k-1}, \alpha \in \mathbb{Z}^{m}\right\}$ to a refined polygon $f^{k}=\left\{f_{\alpha}^{k}, \alpha \in \mathbb{Z}^{m}\right\}$ is defined as

$$
f_{\alpha}^{k}=\sum_{\beta \in \mathbb{Z}^{m}} a_{\alpha-2 \beta} f_{\beta}^{k-1}, \quad \alpha \in \mathbb{Z}^{m} .
$$

The symbol of above subdivision scheme is given by the Laurent polynomial

$$
a(z)=\sum_{\alpha \in \mathbb{Z}^{m}} a_{\alpha} z^{\alpha}, \quad z \in(\mathbb{C} \backslash\{0\})^{m},
$$

where $a=\left\{a_{\alpha}, \alpha \in \mathbb{Z}^{m}\right\}$ is called the mask of subdivision scheme. The detailed information about refinement rules and Laurent polynomial can be found in [1-3].

Surface modeling via subdivision is very important topic in computer graphics and computer aided geometric design and it has been studied by several authors; see surveys [4-10] and references therein.

The construction of univariate subdivision schemes by different variants of Lane-Riesenfeld algorithm has been studied by few authors in $[8,11-13]$ and construction of bivariate subdivision schemes by a variant of Lane-Riesenfeld algorithm has been studied by Romani [8]. In fact, the LaneRiesenfeld algorithm is the combination of two operators. One operator refines the initial points and other operator smooths these refining points for a fixed number of times. Therefore it is also named as Refining-Smooth algorithm (RSalgorithm). Moreover, more smoothing steps provide limit curves/surfaces of wider support as well as of higher smoothness.

Lane and Riesenfeld [7] proposed an algorithm for generating $(n+1)$-degree $\mathrm{B}$-spline curve, which is the simplest form of the RS-algorithm. In Lane-Riesenfeld algorithm, they combined the symbols of linear B-spline scheme and odd 


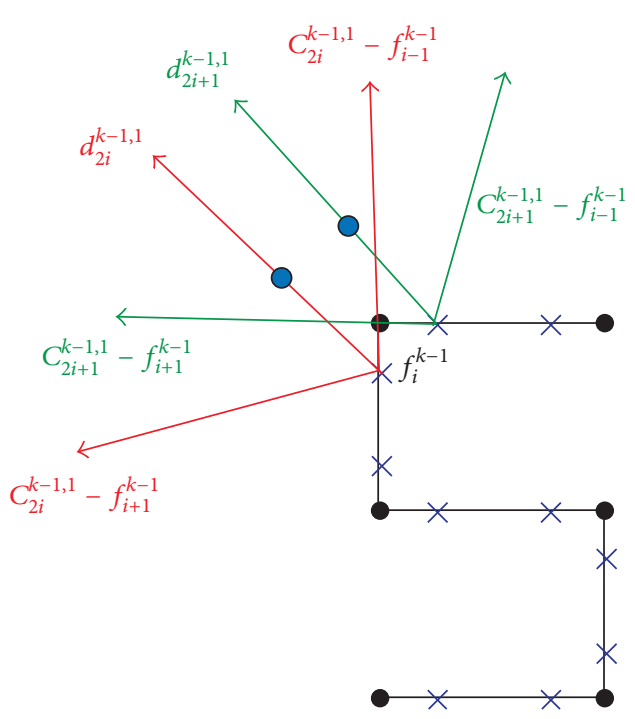

(a) Our refining stage

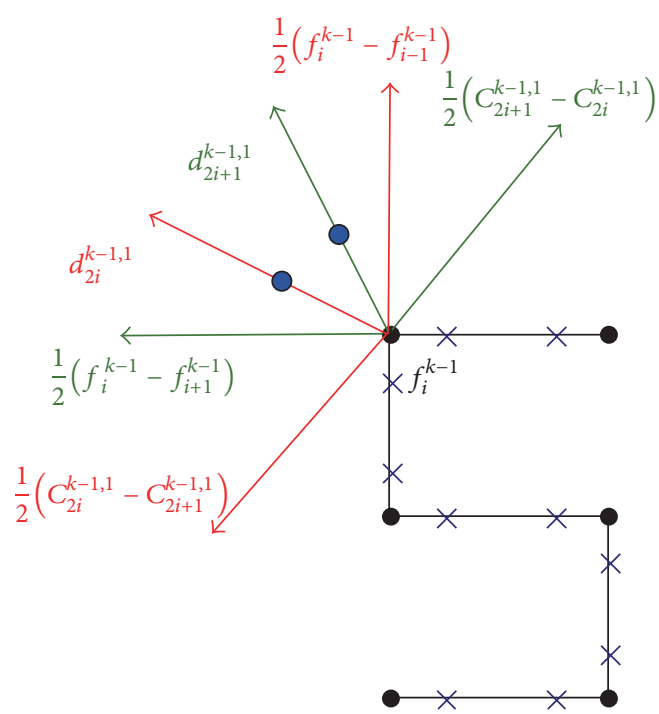

(b) Romani's refining stage

FIGURE 1: The geometrical difference between our refining stage and Romani's refining stage.

stencil of linear B-spline scheme as refining and smoothing operators, respectively. Therefore the symbol of $n$th family member of Lane-Riesenfeld schemes gives symbol of $(n+1)$ degree $\mathrm{B}$-spline scheme. Their family gives $C^{n}$ continuity and linear polynomial reproduction.

In literature, there are few variants which are applied on the Lane-Riesenfeld algorithm. For example, Cashman et al. [12] also proposed a family of univariate subdivision schemes by using the RS-algorithm which is based on Dubuc Deslauriers 4-point interpolatory scheme [14]. They combined the symbols of 4-point interpolatory subdivision scheme and odd stencil of 4-point interpolatory subdivision scheme as refining and smoothing operators, respectively. Their univariate family gives cubic polynomial reproduction but by increasing smoothing stages continuity of their family may or may not be increased.

Ashraf et al. [11] proposed a family of univariate subdivision schemes by using similar technique to that used by Cashman et al. [12] on Dubuc Deslauriers 6-point interpolatory scheme [14]. They also combined the symbol and odd stencil's symbol of Dubuc Deslauriers 6-point interpolatory scheme [14] as refining and smoothing operators, respectively. Their univariate family gives quintic polynomial reproduction but level of continuity of their family does not increase in general by increasing the smoothing stages.

Mustafa et al. [13] also proposed a family of univariate subdivision schemes by using the symbol of 4-point interpolatory subdivision scheme [15] as the refining operator and the symbol of even stencil of the 4-point approximating scheme [16] as the smoothing operator. Their univariate family gives cubic polynomial reproduction but level of continuity of their family also does not increase in general by increasing the smoothing stages.

Romani [8] proposed families of univariate and bivariate subdivision schemes by using RS-algorithm in which the refining operator is based on a perturbation of Chaikin's corner cutting subdivision scheme [17] and the smoothing operator takes the average of two adjacent vertices as in LaneRiesenfeld algorithm. Their univariate family gives linear polynomial reproduction and continuity of their $n$th family member is $C^{n}$. Moreover, if $\omega=1 / 16$, their $n$th family member becomes $(n+3)$ th member of Hormann and Sabin's [16] family and it gives cubic polynomial reproduction.

There are few variants of Lane-Riesenfeld algorithm but all those algorithms started with two binary schemes, by taking the symbol of first scheme as the refining operator and the odd or even symbol of second scheme as the smoothing operator. Our approach is different in the sense that we do not use symbol of any existing subdivision scheme as refining operator and also we choose appropriate smoothing operator by ourselves.

In this paper, we propose a new RS-algorithm which is also a variant of Lane-Riesenfeld algorithm that compared with the one proposed by Romani; we modify refining and smoothing operators both. There are two main differences between our RS-algorithm and Romani's RS-algorithm, which are as follows:

(i) Both refining operators depend on Chaikin points, computed around initial points, but the difference between our refining operator and Romani's refining operator is that Romani draws two vectors at every initial control point and changes position of both Chaikin points, which have been computed around corresponding initial point, by using these two vectors, while we draw one vector at every Chaikin point and change the position of each Chaikin point by using each corresponding vector which we have drawn on it (see Figure 1).

(ii) Difference between both smoothing operators is that Romani's smoothing operator takes average of two 
adjacent points while our smoothing operator takes weighted average of the four adjacent points.

Our refining and smoothing operators are different from the operators proposed by Romani. The framework proposed in this paper provides families of schemes where all members are approximating while the first member of Romani's family is interpolatory and all the other members are approximating. Moreover, the flexibility of our shape parameter allows us to adjust the value of shape parameter locally according to the geometry of initial polygon.

The article is organized as follows. In Section 2, we present the framework for the construction of univariate and bivariate families of subdivision schemes and their graphical and mathematical comparisons with Romani's families. In Section 3, we present some basic properties of these families of subdivision schemes that include discussion on basic limit functions, analysis of continuity, polynomial generation, and reproduction of the schemes. In Section 4, applications of some members of the family and comparisons with Romani schemes are presented. Conclusions are given in Section 5.

\section{Construction and Comparison}

The Refining-Smooth subdivision scheme is the scheme which consists of two operators. One operator refines the initial points while the other operator smooths the refined points for the required number of times. If we denote refining operator by $R$, smoothing operator by $S$, and the subdivision operator by $S_{a_{l}}$, then

$$
f^{k}=S_{a_{l}} f^{k-1}
$$

where

$$
\begin{aligned}
f^{k-1,1} & =R f^{k-1}, \\
f^{k} & =f^{k-1, n+1}=S f^{k-1, n}, \quad n=1,2, \ldots, l .
\end{aligned}
$$

In fact, refining operator $R$ maps a polygon $f^{k-1}=$ $\left\{f_{\alpha}^{k-1}, \alpha \in \mathbb{Z}^{m}\right\}$ to a refined polygon $f^{k-1,1}=\left\{f_{\alpha}^{k-1,1}, \alpha \in \mathbb{Z}^{m}\right\}$, where $m=1,2$; that is,

$$
f_{\alpha}^{k-1,1}=\sum_{\beta \in \mathbb{Z}^{m}} r_{\alpha-2 \beta} f_{\beta}^{k-1}, \quad \alpha \in \mathbb{Z}^{m},
$$

and smoothing operator $S$ maps a polygon $f^{k-1, n}=$ $\left\{f_{\alpha}^{k-1, n}, \alpha \in \mathbb{Z}^{m}\right\}$ to a refined polygon $f^{k-1, n+1}=\left\{f_{\alpha}^{k-1, n+1}, \alpha \in\right.$ $\left.\mathbb{Z}^{m}\right\}$ which is defined as

$$
f_{\alpha}^{k-1, n+1}=\sum_{\beta \in \mathbb{Z}^{m}} s_{\beta} f_{\beta}^{k-1, n}, \quad \alpha \in \mathbb{Z}^{m}, n=1,2, \ldots, l .
$$

The symbols of refining and smoothing operators are

$$
\begin{aligned}
& r(z)=\sum_{\alpha \in \mathbb{Z}^{m}} r_{\alpha} z^{\alpha}, \\
& s(z)=\sum_{\alpha \in \mathbb{Z}^{m}} s_{\alpha} z^{\alpha}, \\
& z \in(\mathbb{C} \backslash\{0\})^{m},
\end{aligned}
$$

with

$$
\begin{aligned}
& r(\mathbf{1})=2^{m}, \\
& s(\mathbf{1})=1,
\end{aligned}
$$

where $\mathbf{1} \in \mathbb{R}^{m}$.

In RS-algorithm, combination of one refining operator and $l$ smoothing operators is called the subdivision operator. Moreover, we denote proposed univariate and bivariate families of subdivision schemes by $S_{a_{l}}$ and $S_{b_{l}}$, respectively.

The construction procedure for our families of schemes is given below.

2.1. Univariate Family. Here we denote the Chaikin points by $C_{2 i}^{k-1,1}$ and $C_{2 i+1}^{k-1,1}$ that are computed around the point $f_{i}^{k-1,0}=$ $f_{i}^{k-1}$ as shown in Figure 1. In this figure, black solid circles show control points at $(k-1)$ th subdivision level, blue crosses show the Chaikin points, and blue solid circles show refining points. Red vectors are involved in the insertion of even point $f_{2 i}^{k-1,1}$ and green vectors are involved in the insertion of odd point $f_{2 i+1}^{k-1,1}$. In this figure, we have shown the refinement of only two Chaikin points which have been inserted around point $f_{i}^{k-1}$.

In our framework, we compute vectors $d_{2 i}^{k-1,1}$ and $d_{2 i+1}^{k-1,1}$ by taking the linear combinations of two vectors, as shown in Figure 1(a):

$$
\begin{aligned}
d_{2 i}^{k-1,1}= & (l+1)\left(C_{2 i}^{k-1,1}-f_{i+1}^{k-1}\right) \\
& +(l-1)\left(C_{2 i}^{k-1,1}-f_{i-1}^{k-1}\right), \\
d_{2 i+1}^{k-1,1}= & (l+1)\left(C_{2 i+1}^{k-1,1}-f_{i-1}^{k-1}\right) \\
& +(l-1)\left(C_{2 i+1}^{k-1,1}-f_{i+1}^{k-1}\right),
\end{aligned}
$$

where in Figure 1(b) vectors are computed as

$$
\begin{aligned}
d_{2 i}^{k-1,1}= & (l+3)\left(C_{2 i}^{k-1,1}-C_{2 i+1}^{k-1,1}\right) \\
& +\left(\frac{l-1}{2}\right)\left(f_{i}^{k-1}-f_{i-1}^{k-1}\right), \\
d_{2 i+1}^{k-1,1}= & (l+3)\left(C_{2 i+1}^{k-1,1}-C_{2 i}^{k-1,1}\right) \\
& +\left(\frac{l-1}{2}\right)\left(f_{i}^{k-1}-f_{i+1}^{k-1}\right),
\end{aligned}
$$

with $l \in \mathbb{N}$.

In our framework, we compute refining point by adding each Chaikin point to the corresponding vector; that is,

$$
\begin{aligned}
& f_{2 i}^{k-1,1}=C_{2 i}^{k-1,1}+\omega d_{2 i}^{k-1,1} \\
& f_{2 i+1}^{k-1,1}=C_{2 i+1}^{k-1,1}+\omega d_{2 i+1}^{k-1,1}
\end{aligned}
$$




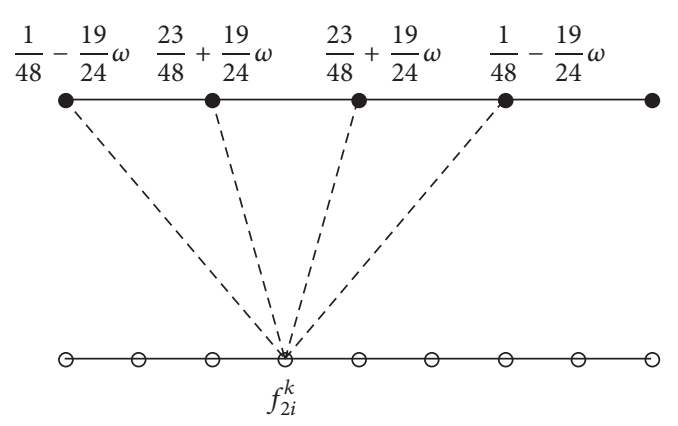

(a) Edge rule

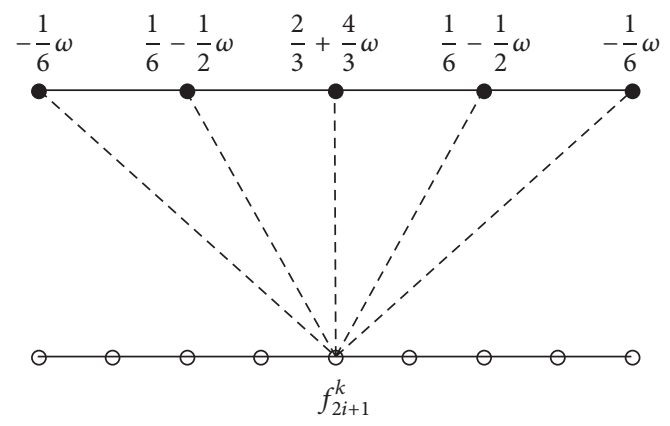

(b) Vertex rule

Figure 2: Schematic overview of the refinement rules when we apply one time refining operator for $l=1$ and one time smoothing operator. Solid circles show the points at $(k-1)$ th subdivision level while circles show the points at $k$ th subdivision level after applying one refining and one smoothing stage.

where $\omega \in \mathbb{R}$. While in Romani's framework refining points are computed as

$$
\begin{aligned}
& f_{2 i}^{k-1,1}=f_{i}^{k-1}+2 \omega d_{2 i}^{k-1,1}, \\
& f_{2 i+1}^{k-1,1}=f_{i}^{k-1}+2 \omega d_{2 i+1}^{k-1,1} .
\end{aligned}
$$

Now by substituting (9) in (11), we get the following subdivision scheme which will refine the course polygon $f^{k-1,0}$ into refined polygon $f^{k-1,1}$ :

$$
\begin{aligned}
& f_{2 i}^{k-1,1}=\alpha_{1} f_{i-1}^{k-1}+\alpha_{2} f_{i}^{k-1}+\alpha_{3} f_{i+1}^{k-1}, \\
& f_{2 i+1}^{k-1,1}=\alpha_{3} f_{i-1}^{k-1}+\alpha_{2} f_{i}^{k-1}+\alpha_{1} f_{i+1}^{k-1},
\end{aligned}
$$

where

$$
\begin{aligned}
& \alpha_{1}=\frac{1}{4}(1+2 \omega(2-l)), \\
& \alpha_{2}=\frac{3}{4}(1+2 \omega l), \\
& \alpha_{3}=-\omega(l+1) .
\end{aligned}
$$

Now the second step in our frame work is to smooth the refined polygon $f^{k-1,1}=\left\{f_{i}^{k-1,1}\right\}_{i \in \mathbb{Z}}$ by applying $l$-times smoothing operator. That is, smoothing operator maps $f_{i}^{k-1,1}$ into $f_{i}^{k}=f_{i}^{k, 0}=f_{i}^{k-1, l+1}$ by using the following rule:

$$
\begin{aligned}
f_{i}^{k-1, n+1}= & \frac{1}{12} f_{i-1}^{k-1, n}+\frac{5}{12} f_{i}^{k-1, n}+\frac{5}{12} f_{i+1}^{k-1, n} \\
& +\frac{1}{12} f_{i+2}^{k-1, n}
\end{aligned}
$$

where $n=1,2, \ldots, l$.
By applying one time refining operator and $l$-times smoothing operator, we get the new family of subdivision schemes with symbol

$$
\begin{aligned}
a_{l}(z) & =z^{-l-[l / 2\rceil-2}\left(\frac{1}{12}(z+1)\left(z^{2}+4 z+1\right)\right)^{l} \\
\cdot & \left(-\omega(l+1)+\frac{1}{4}\{1-2 \omega(l-2)\} z\right. \\
+ & \frac{3}{4}(1+2 \omega l) z^{2}+\frac{3}{4}(1+2 \omega l) z^{3} \\
+ & \left.\frac{1}{4}\{1-2 \omega(l-2)\} z^{4}-\omega(l+1) z^{5}\right),
\end{aligned}
$$

where \lceil\rceil is a ceiling function.

The first member of our proposed family is a primal 5point relaxed subdivision scheme with two refinement rules. The brief description of these refinement rules is given below.

(i) Edge splitting rule: for every edge in the coarse polygon, a new edge point is calculated by using the affine combination of 4 neighboring control points as shown in Figure 2(a).

(ii) Vertex updating rule: for every vertex in the coarse polygon, a new vertex point is calculated by using the affine combination of 5 neighboring control points as shown in Figure 2(b).

Remark 1. For $\omega=0$, symbol (16) takes the form

$$
a_{l}(z)=z^{-l-[l / 2]-1}\left(\frac{z^{2}+4 z+1}{3}\right)^{l} \frac{(z+1)^{l+3}}{4^{l+1}} .
$$

Now we shall construct family of bivariate nontensor product subdivision schemes for quadrilateral meshes by choosing Doo-Sabin points as origin.

2.2. Bivariate Family. Let us denote the Doo-Sabin points for one face of quadrilateral mesh by $C_{2 i, 2 j}^{k-1,1}, C_{2 i+1,2 j}^{k-1,1}, C_{2 i, 2 j+1}^{k-1,1}$, 


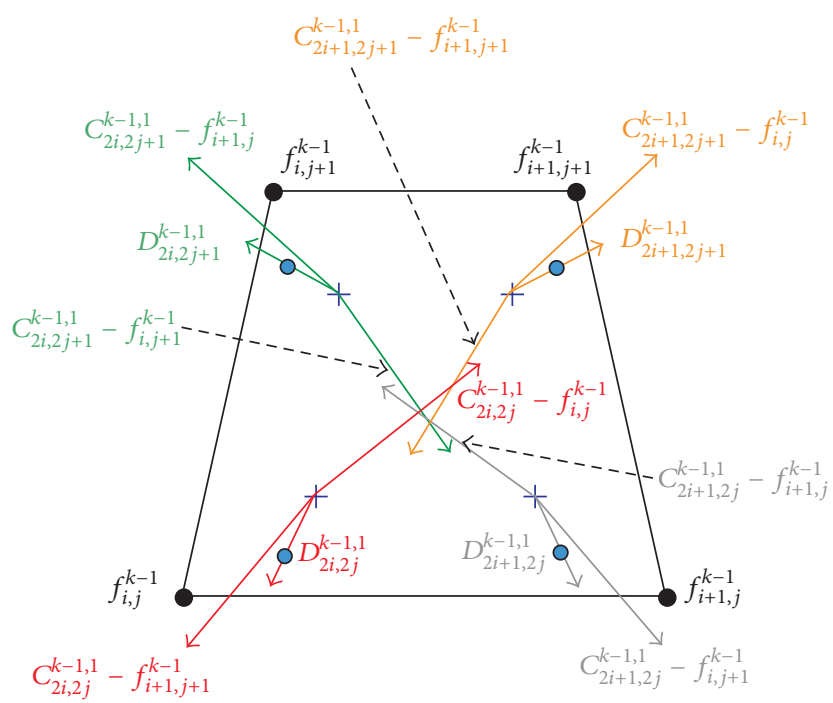

FIgURE 3: The geometrical construction of the refining stage for quadrilateral meshes.

and $C_{2 i+1,2 j+1}^{k-1,1}$ that are computed from the initial points $f_{i, j}^{k-1}$, $f_{i+1, j}^{k-1}, f_{i, j+1}^{k-1}$, and $f_{i+1, j+1}^{k-1}$ as shown in Figure 3. In this figure, black solid circles show initial points at $(k-1)$ th subdivision level, blue cross symbols show Doo-Sabin points, and blue solid circles show refining points at $(k-1)$ th level; red, gray, green, and orange vectors are involved in the insertion of the refining points $f_{2 i, 2 j}^{k-1,1}, f_{2 i+1,2 j}^{k-1,1}, f_{2 i, 2 j+1}^{k-1,1}$, and $f_{2 i+1,2 j+1}^{k-1,1}$, respectively.

We compute vectors $D_{2 i, 2 j}^{k-1,1}, \quad D_{2 i+1,2 j}^{k-1,1}, \quad D_{2 i, 2 j+1}^{k-1,1}$, and $D_{2 i+1,2 j+1}^{k-1,1}$ by taking the linear combination of two vectors that are drawn on the Doo-Sabin points $C_{2 i, 2 j}^{k-1,1}, C_{2 i+1,2 j}^{k-1,1}$, $C_{2 i, 2 j+1}^{k-1,1}$, and $C_{2 i+1,2 j+1}^{k-1,1}$, respectively, as shown in Figure 3 .

$$
\begin{aligned}
D_{2 i, 2 j}^{k-1,1}= & (l+1)\left(C_{2 i, 2 j}^{k-1,1}-f_{i+1, j+1}^{k-1}\right) \\
& +(l-1)\left(C_{2 i, 2 j}^{k-1,1}-f_{i, j}^{k-1}\right), \\
D_{2 i+1,2 j}^{k-1,1}= & (l+1)\left(C_{2 i+1,2 j}^{k-1,1}-f_{i, j+1}^{k-1}\right) \\
& +(l-1)\left(C_{2 i+1,2 j}^{k-1,1}-f_{i+1, j}^{k-1}\right), \\
D_{2 i, 2 j+1}^{k-1,1}= & (l+1)\left(C_{2 i, 2 j+1}^{k-1,1}-f_{i+1, j}^{k-1}\right) \\
& +(l-1)\left(C_{2 i, 2 j+1}^{k-1,1}-f_{i, j+1}^{k-1}\right), \\
D_{2 i+1,2 j+1}^{k-1,1}= & (l+1)\left(C_{2 i+1,2 j+1}^{k-1,1}-f_{i, j}^{k-1}\right) \\
& +(l-1)\left(C_{2 i+1,2 j+1}^{k-1,1}-f_{i+1, j+1}^{k-1}\right),
\end{aligned}
$$

where $l \in \mathbb{N}$.

Similarly to the curve case, we compute refining points as

$$
\begin{gathered}
f_{2 i, 2 j}^{k-1,1}=C_{2 i, 2 j}^{k-1,1}+\omega D_{2 i, 2 j}^{k-1,1}, \\
f_{2 i+1,2 j}^{k-1,1}=C_{2 i+1,2 j}^{k-1,1}+\omega D_{2 i+1,2 j}^{k-1,1}
\end{gathered}
$$

$$
\begin{aligned}
f_{2 i, 2 j+1}^{k-1,1} & =C_{2 i, 2 j+1}^{k-1,1}+\omega D_{2 i, 2 j+1}^{k-1,1}, \\
f_{2 i+1,2 j+1}^{k-1,1} & =C_{2 i+1,2 j+1}^{k-1,1}+\omega D_{2 i+1,2 j+1}^{k-1,1}
\end{aligned}
$$

where $\omega \in \mathbb{R}$.

Now by substituting (18) in (19), we get the subdivision scheme which will refine the course mesh $\mathbf{f}^{\mathbf{k}-\mathbf{1 , 0}}$ into refined mesh $\mathbf{f}^{\mathrm{k}-\mathbf{1 , 1}}$.

$$
\begin{aligned}
f_{2 i, 2 j}^{k-1,1} & =\beta_{1} f_{i, j}^{k-1}+\beta_{2} f_{i+1, j}^{k-1}+\beta_{2} f_{i, j+1}^{k-1}+\beta_{3} f_{i+1, j+1}^{k-1}, \\
f_{2 i+1,2 j}^{k-1,1} & =\beta_{2} f_{i, j}^{k-1}+\beta_{1} f_{i+1, j}^{k-1}+\beta_{3} f_{i, j+1}^{k-1}+\beta_{2} f_{i+1, j+1}^{k-1}, \\
f_{2 i, 2 j+1}^{k-1,1} & =\beta_{2} f_{i, j}^{k-1}+\beta_{3} f_{i+1, j}^{k-1}+\beta_{1} f_{i, j+1}^{k-1}+\beta_{2} f_{i+1, j+1}^{k-1}, \\
f_{2 i+1,2 j+1}^{k-1,1} & =\beta_{3} f_{i, j}^{k-1}+\beta_{2} f_{i+1, j}^{k-1}+\beta_{2} f_{i, j+1}^{k-1}+\beta_{1} f_{i+1, j+1}^{k-1},
\end{aligned}
$$

where $\beta_{1}=(1 / 16)\{9+2(l+8) \omega\}, \beta_{2}=(1 / 16)\{3(2 \omega l+1)\}$, and $\beta_{3}=(1 / 16)\{1-2(7 l+8) \omega\}$.

Now we smooth the refined mesh $\mathbf{f}^{\mathbf{k}-\mathbf{1 , 1}}=\left\{f_{i, j}^{k-1,1}\right\}_{i, j \in \mathbb{Z}}$ by applying $l$-time smoothing operator. That is, we map $f_{i, j}^{k-1,1}$ into $f_{i, j}^{k}=f_{i, j}^{k, 0}=f_{i, j}^{k-1, l+1}$ by using the following smoothing operator:

$$
\begin{gathered}
f_{i, j}^{k-1, n+1}=\frac{1}{12}\left\{\frac{1}{12} f_{i-1, j-1}^{k-1, n}+\frac{5}{12} f_{i, j-1}^{k-1, n}+\frac{5}{12} f_{i+1, j-1}^{k-1, n}\right. \\
\left.+\frac{1}{12} f_{i+2, j-1}^{k-1, n}\right\}+\frac{5}{12}\left\{\frac{1}{12} f_{i-1, j}^{k-1, n}+\frac{5}{12} f_{i, j}^{k-1, n}\right. \\
\left.+\frac{5}{12} f_{i+1, j}^{k-1, n}+\frac{1}{12} f_{i+2, j}^{k-1, n}\right\}+\frac{5}{12}\left\{\frac{1}{12} f_{i-1, j+1}^{k-1, n}\right. \\
\left.+\frac{5}{12} f_{i, j+1}^{k-1, n}+\frac{5}{12} f_{i+1, j+1}^{k-1, n}+\frac{1}{12} f_{i+2, j+1}^{k-1, n}\right\}
\end{gathered}
$$




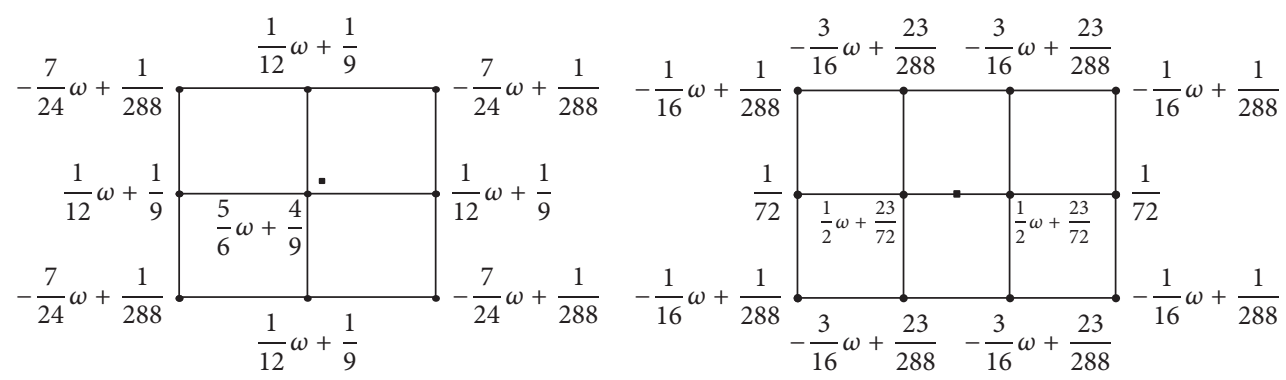

(a) Vertex rule

(b) Edge rule

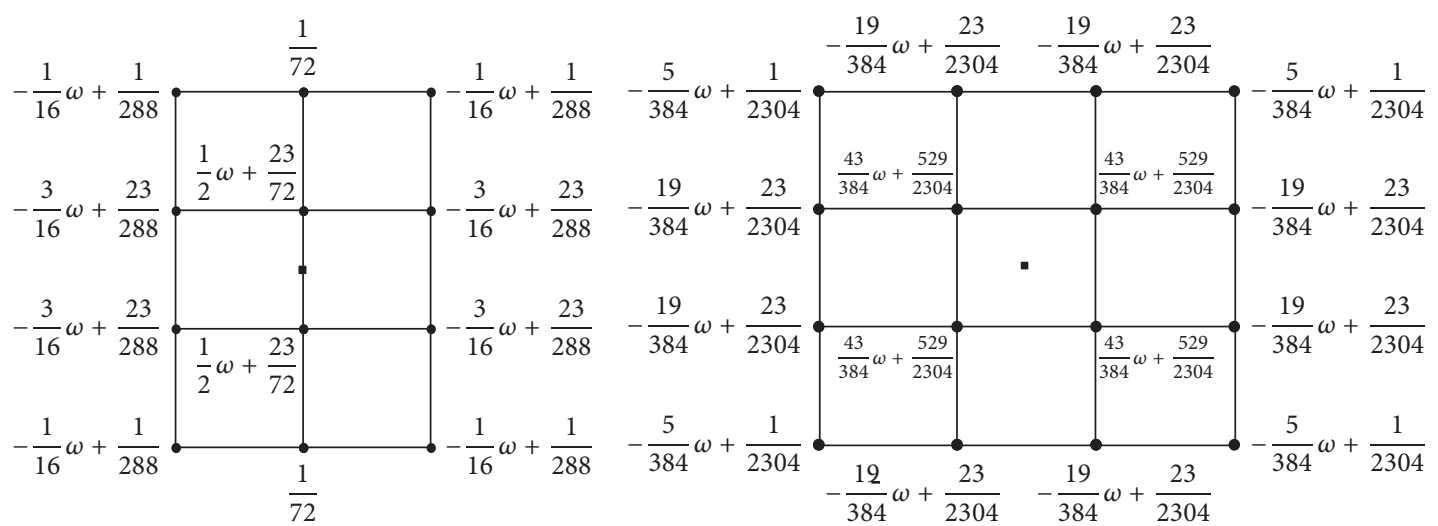

(c) Edge rule

(d) Face rule

FIGURE 4: Schematic overview of the refinement rules for $l=1$. Solid circles show points at $(k-1)$ th subdivision level where solid squares show the new points at level $k$.

$$
\begin{aligned}
& +\frac{1}{12}\left\{\frac{1}{12} f_{i-1, j+2}^{k-1, n}+\frac{5}{12} f_{i, j+2}^{k-1, n}+\frac{5}{12} f_{i+1, j+2}^{k-1, n}\right. \\
& \left.+\frac{1}{12} f_{i+2, j+2}^{k-1, n}\right\}
\end{aligned}
$$

where $n=1,2, \ldots, l$. By applying one time refining operator $R$ and $l$-times smoothing operator $S$, we get the family of bivariate primal/dual subdivision schemes for quadrilateral meshes with symbol

$$
\begin{aligned}
b_{l}\left(z_{1}, z_{2}\right)=\left(z_{1} z_{2}\right)^{-1-l-\lceil l / 2\rceil}\left(\frac{1}{144}\left(z_{2}+1\right)\right. \\
\left.\cdot\left(z_{2}^{2}+4 z_{2}+1\right)\left(z_{1}+1\right)\left(z_{1}^{2}+4 z_{1}+1\right)\right)^{l} \\
\cdot\left[\frac{1}{16}\{9+2(l+8) \omega\} z_{1} z_{2}+\frac{1}{16}\{3(1+2 \omega l)\} z_{1}^{3} z_{2}\right. \\
+\frac{1}{16}\{3(1+2 \omega l)\} z_{1} z_{2}^{3}+\frac{1}{16}\{1-2(7 l+8) \omega\} \\
\cdot z_{1}^{3} z_{2}^{3}+\frac{1}{16}\{3(1+2 \omega l)\} z_{2}+\frac{1}{16}\{9+2(l+8) \omega\} \\
\cdot z_{1}^{2} z_{2}+\frac{1}{16}\{1-2(7 l+8) \omega\} z_{2}^{3}+\frac{1}{16}\{3(1+2 \omega l)\} \\
\cdot z_{1}^{2} z_{2}^{3}+\frac{1}{16}\{3(1+2 \omega l)\} z_{1}+\frac{1}{16}\{1-2(7 l+8) \omega\}
\end{aligned}
$$

$$
\begin{aligned}
& \cdot z_{1}^{3}+\frac{1}{16}\{9+2(l+8) \omega\} z_{1} z_{2}^{2}+\frac{1}{16}\{3(1+2 \omega l)\} \\
& \cdot z_{1}^{3} z_{2}^{2}+\frac{1}{16}\{1-2(7 l+8) \omega\}+\frac{1}{16}\{3(1+2 \omega l)\} z_{1}^{2} \\
& \left.+\frac{1}{16}\{3(1+2 \omega l)\} z_{2}^{2}+\frac{1}{16}\{9+2(l+8) \omega\} z_{1}^{2} z_{2}^{2}\right] .
\end{aligned}
$$

Remark 2. The bivariate family for $\omega=0$ corresponding to the symbol (22) reduces to the tensor product univariate family. This tensor product family of schemes can also be obtained from the symbol defined by (16). If we apply one time refining operator (20) for $l=1$ and one time smoothing operator (21), we get the primal approximating subdivision scheme with four refinement rules. The schematic overview of these refinement rules is given in Figure 4.

Remark 3. For $z_{1}=1$ and $z_{2}=z$ or $z_{1}=z$ and $z_{2}=1$, our family of bivariate schemes reduce to the family of univariate schemes with the symbol

$$
\begin{aligned}
& b_{l}(z)=\frac{1}{2} b_{l}(1, z)=\frac{1}{2} b_{l}(z, 1)=z^{-(l+1)-\lceil l / 2\rceil} \\
& \cdot \frac{(1+z)^{l+1}}{2^{l+2}}\left(\frac{z^{2}+4 z+1}{6}\right)^{l}\left[\{1-2(l+2) \omega\} z^{2}\right. \\
& +2\{1+2(l+2) \omega\} z+\{1-2(l+2) \omega\}] .
\end{aligned}
$$


This family of schemes is different from our proposed univariate family of schemes.

\section{Analysis of Univariate and Bivariate Schemes}

This section deals with the analysis of proposed families of schemes. The analysis includes support width and smoothness/continuity via Laurent polynomial method. The capacity of generating and reproducing polynomials has also been discussed mathematically.

3.1. Support of Basic Limit Function. Support of basic limit function of a subdivision scheme is equal to the number of spans of the curve affected when one control point is moved from its initial place.

A convergent subdivision scheme $S_{a_{l}}$ defines a basic limit function $\phi_{l}=S_{a_{l}}^{\infty} \delta_{i}$ with $\delta_{i}$ being the initial data such that

$$
\delta_{i}= \begin{cases}1 & \text { for } i=0 \\ 0 & \text { otherwise }\end{cases}
$$

Support of basic limit function of our univariate family of schemes depends on parameter $\omega$.

(i) For $\omega \neq 0 \in \Omega_{l}$, the numbers of nonzero coefficients in the symbol of refining operator (13) and smoothing operator (15) are 6 and 4, respectively. So by [18] support size of basic limit function of the schemes $S_{a_{l}}$ for the general symbol $a_{l}(z)$ is $3 l+5$ and its support width is $[-(3 l+5) / 2,(3 l+5) / 2]$.

(ii) For $\omega=0$, the number of nonzero coefficients in the symbol of both refining and smoothing stages is 4 ; therefore support size of basic limit function of the schemes $S_{a_{l}}$ for the general symbol $a_{l}(z)$ is $3(l+1)$ and its support width is $[-(3 / 2)(l+1),(3 / 2)(l+1)]$.

A convergent bivariate subdivision scheme $S_{b_{l}}$ defines a basic limit function $\phi_{l}=S_{b_{l}}^{\infty} \delta_{i, j}$, where $\delta_{i, j}$ is the initial data such that

$$
\delta_{i, j}= \begin{cases}1 & \text { for } i=j=0 \\ 0 & \text { otherwise }\end{cases}
$$

Support of basic limit of our bivariate family of schemes is $3(l+1) \times 3(l+1)$ and its support width is $[-(3 / 2)(l+1),(3 / 2)(l+$ $1)] \times[-(3 / 2)(l+1),(3 / 2)(l+1)]$.

Basic limit functions for the first three family members of univariate family for three different values of shape parameters are presented in Figure 5, whereas basic limit functions for the first family member of bivariate family for three different values of shape parameter are presented in Figure 6. From these figures, we see that the support width for positive value is less than the support width for negative value of the parameter involved in the bivariate family.
3.2. Continuity Analysis. Here we present the smoothness of proposed families of schemes by using the Laurent polynomial method. For this purpose, we have used the methods that are given ([3], Theorems 4.11, 4.13, 4.29, and 4.30).

Theorem 4. The subdivision scheme $S_{a_{1}}$ is convergent for $-7 /$ $8<\omega<13 / 38$, generates $C^{1}$ limiting curve for $-3 / 10<\omega<$ $1 / 6$, and generates $C^{3}$ limiting curve for $\omega=0$.

Proof. Since the scheme with the symbol $(z /(1+z)) a_{1}(z)$ is contractive for $-7 / 8<\omega<13 / 38$, therefore the scheme $S_{a_{1}}$ is convergent for $-7 / 8<\omega<13 / 38$. Similarly, the scheme with the symbol $2(z /(1+z))^{2} a_{1}(z)$ is contractive for $-3 / 10<$ $\omega<1 / 6$; this implies that the scheme $S_{a_{1}}$ is $C^{1}$ continuous for $-3 / 10<\omega<1 / 6$. Moreover, the scheme with symbol $4(z /(1+$ $z))^{3} a_{1}(z)$ is contractive for $\omega=0$; accordingly the scheme $S_{a_{1}}$ is $C^{2}$ continuous for $\omega=0$. Furthermore, the scheme with symbol $6(z /(1+z))^{4} a_{1}(z)$ is contractive for $\omega=0$; hence the scheme $S_{a_{1}}$ is $C^{3}$ continuous for $\omega=0$.

Theorem 5. (i) The subdivision scheme $S_{a_{2}}$ is convergent for $-53 / 100<\omega<7 / 26$, generates $C^{1}$ and $C^{2}$ limiting curves for $-53 / 100<\omega<7 / 26$ and $-11 / 27<\omega<19 / 108$, respectively, and generates $C^{4}$ limiting curve for $\omega=0$.

(ii) The subdivision scheme $S_{a_{3}}$ is convergent for $-1723 /$ $3322<\omega<585 / 1954$, generates $C^{1}, C^{2}$, and $C^{3}$ limiting curves for $-114 / 271<\omega<269 / 1154,-114 / 271<\omega<269 /$ 1154 , and $-19 / 58<\omega<61 / 358$, respectively, and generates $C^{5}$ limiting curve for $\omega=0$.

(iii) The subdivision scheme $S_{a_{4}}$ is convergent for -5021/ $11121<\omega<2099 / 7808$, generates $C^{1}, C^{2}, C^{3}$, and $C^{4}$ limiting curves for $-5021 / 11121<\omega<2099 / 7808,-2635 / 7128<$ $\omega<854 / 3993,-2635 / 7128<\omega<854 / 3993$, and $-703 /$ $2404<\omega<391 / 2384$, respectively, and generates $C^{6}$ limiting curve for $\omega=0$.

Proof. Proof of this theorem is same as Theorem 4.

Theorem 6. The subdivision scheme $S_{b_{1}}$ is convergent for $-41 / 36<\omega<11 / 21$, generates $C^{1}$ limiting surface for $-1 / 2<$ $\omega<19 / 45$, and generates $C^{3}$ limiting surface for $\omega=0$.

Proof. Since the schemes with symbols $\left(z_{1} /\left(1+z_{1}\right)\right) b_{1}\left(z_{1}, z_{2}\right)$ and $\left(z_{2} /\left(1+z_{2}\right)\right) b_{1}\left(z_{1}, z_{2}\right)$ are contractive for $-41 / 36<\omega<$ $11 / 21$, therefore the scheme $S_{b_{1}}$ is convergent for $-41 / 36<$ $\omega<11 / 21$. Similarly, the schemes with symbols $2\left(z_{1} /(1+\right.$ $\left.\left.z_{1}\right)\right)^{2} b_{1}\left(z_{1}, z_{2}\right)$ and $2\left(z_{2} /\left(1+z_{2}\right)\right)^{2} b_{1}\left(z_{1}, z_{2}\right)$ are contractive for $-1 / 2<\omega<5 / 18$ and the scheme with symbol $2\left(z_{1} /(1+\right.$ $\left.\left.z_{1}\right)\right)\left(z_{2} /\left(1+z_{2}\right)\right) b_{1}\left(z_{1}, z_{2}\right)$ is contractive for $-17 / 15<\omega<$ $19 / 45$; this implies that the scheme $S_{b_{1}}$ is $C^{1}$ for $-1 / 2<$ $\omega<19 / 45$. Moreover, the schemes with symbols $4\left(z_{1} /(1+\right.$ $\left.\left.z_{1}\right)\right)^{3} b_{1}\left(z_{1}, z_{2}\right), 4\left(z_{2} /\left(1+z_{2}\right)\right)^{3} b_{1}\left(z_{1}, z_{2}\right), 4\left(z_{1} /\left(1+z_{1}\right)\right)^{2}\left(z_{2} /(1+\right.$ $\left.\left.z_{2}\right)\right) b_{1}\left(z_{1}, z_{2}\right)$, and $4\left(z_{1} /\left(1+z_{1}\right)\right)\left(z_{2} /\left(1+z_{2}\right)\right)^{2} b_{1}\left(z_{1}, z_{2}\right)$ are contractive for $\omega=0$; accordingly the scheme $S_{b_{1}}$ is $C^{2}$ for $\omega=0$. Furthermore, the schemes with symbols $6\left(z_{1} /(1+\right.$ $\left.\left.z_{1}\right)\right)^{4} b_{1}\left(z_{1}, z_{2}\right), 6\left(z_{2} /\left(1+z_{2}\right)\right)^{4} b_{1}\left(z_{1}, z_{2}\right), 6\left(z_{1} /\left(1+z_{1}\right)\right)^{3}\left(z_{2} /(1+\right.$ $\left.\left.z_{2}\right)\right) \times b_{1}\left(z_{1}, z_{2}\right), 6\left(z_{1} /\left(1+z_{1}\right)\right)\left(z_{2} /\left(1+z_{2}\right)\right)^{3} b_{1}\left(z_{1}, z_{2}\right)$, and 


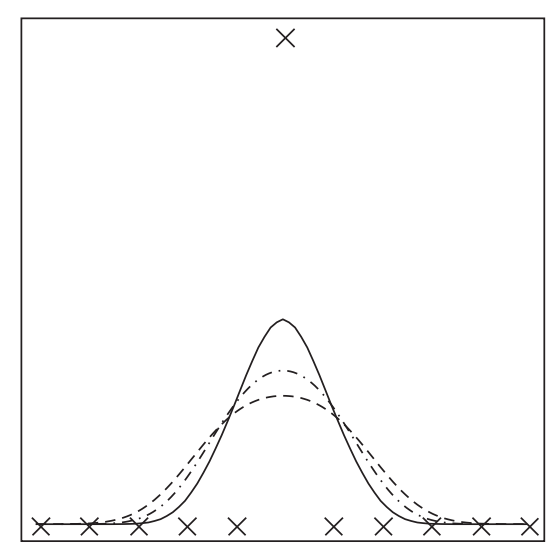

(a) $\omega=-1 / 8$

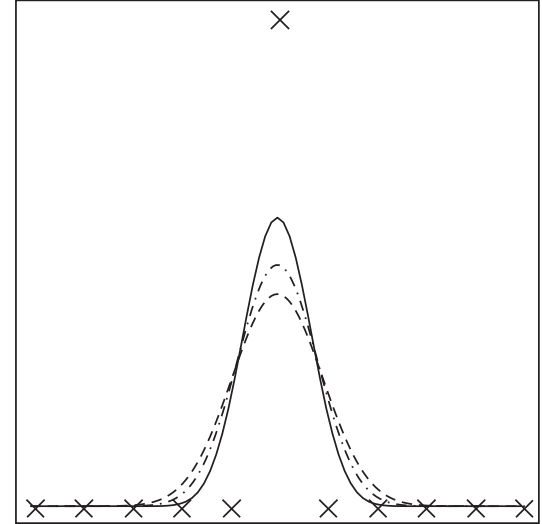

(b) $\omega=0$

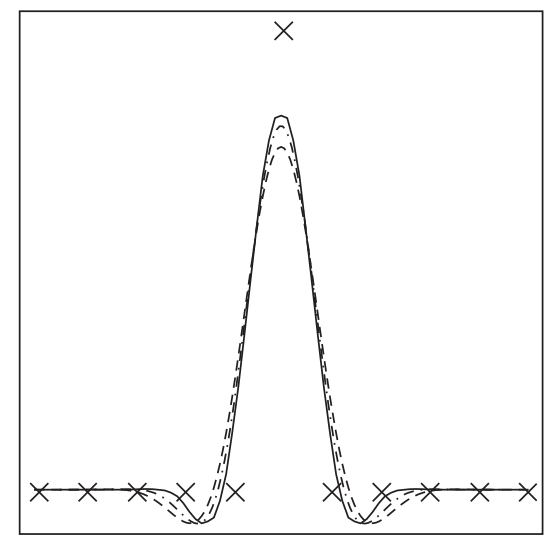

(c) $\omega=1 / 8$

Figure 5: Basic limit functions of subdivision schemes $S_{a_{l}}$ for three values of shape parameter $\omega$. Cross symbols show control points, while solid lines, dashed dotted lines, and dash lines represent curves obtained by subdivision schemes $S_{a_{1}}, S_{a_{2}}$, and $S_{a_{3}}$, respectively.

$6\left(z_{1} /\left(1+z_{1}\right)\right)^{2}\left(z_{2} /\left(1+z_{2}\right)\right)^{2} b_{1}\left(z_{1}, z_{2}\right)$ are contractive for $\omega=0$ thus the scheme $S_{b_{1}}$ is $C^{3}$ for $\omega=0$.

Theorem 7. The subdivision scheme $S_{b_{2}}$ is convergent for $-14075 / 12444<\omega<1191 / 1600$, generates $C^{1}$ and $C^{2}$ limiting surfaces for $-5463 / 4928<\omega<801 / 1388$ and $-11 / 16<\omega<1089 / 2716$, respectively, and generates $C^{4}$ limiting surface for $\omega=0$.

Proof. Proof is same as Theorem 6.

When the initial control points are taken at the same intervals from some polynomial and if the new data points after applying subdivision scheme are located on the polynomial of same degree, then we say that the subdivision scheme generates polynomials up to that certain degree (degree of polynomial). But if the new data points after applying subdivision scheme are located on the same polynomial, then we say that the subdivision scheme reproduces polynomials up to that certain degree. See [16] for details. We will discuss the polynomial generation and reproduction of our families in the upcoming subsections.
3.3. Generation Degree. Let $\Omega_{l}$ and $\Omega_{l}^{\prime}$ be the range of $\omega$ for which the subdivision schemes $S_{a_{l}}$ and $S_{b_{l}}$ are convergent, respectively.

Here we compute the polynomial generation of our proposed family of schemes by using the conditions given in [1] (Conditions $Z_{1}$ and $Z_{k}$ ).

Theorem 8. The subdivision scheme $S_{a_{l}}$ generates polynomials

(i) up to degree $l$ for $\omega \in \Omega_{l}$,

(ii) up to degree $l+2$ for $\omega=0$.

Proof. Since $a_{l}(1)=2, a_{l}(-1)=0$, and for $\omega \in \Omega_{l}$, $\left.D^{(n)} a_{l}(z)\right|_{z=-1}=0, n=1,2,3, \ldots, l$. Then by [1] (Condition $\left.Z_{k}\right), S_{a_{l}}$ generates polynomial up to degree $l$. For $\omega=0$, $\left.D^{(n+2)} a_{l}(z)\right|_{z=-1}=0, n=1,2,3, \ldots, l$. Therefore again by [1] (Condition $Z_{k}$ ), $S_{a_{l}}$ generates polynomial up to degree $l+2$.

Theorem 9. The subdivision scheme $S_{b_{1}}$ generates

(i) linear polynomials for $\omega \in \Omega_{1}^{\prime}$,

(ii) cubic polynomials for $\omega=0$. 


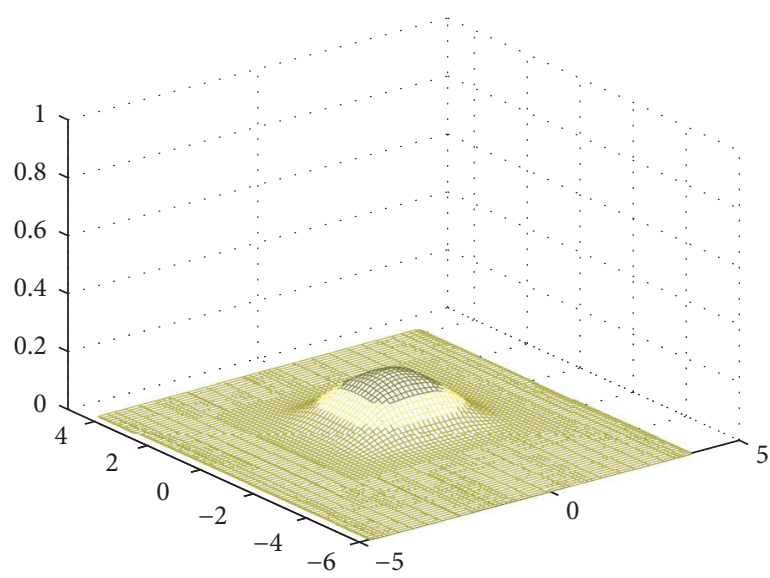

(a) $\omega=-1 / 3$

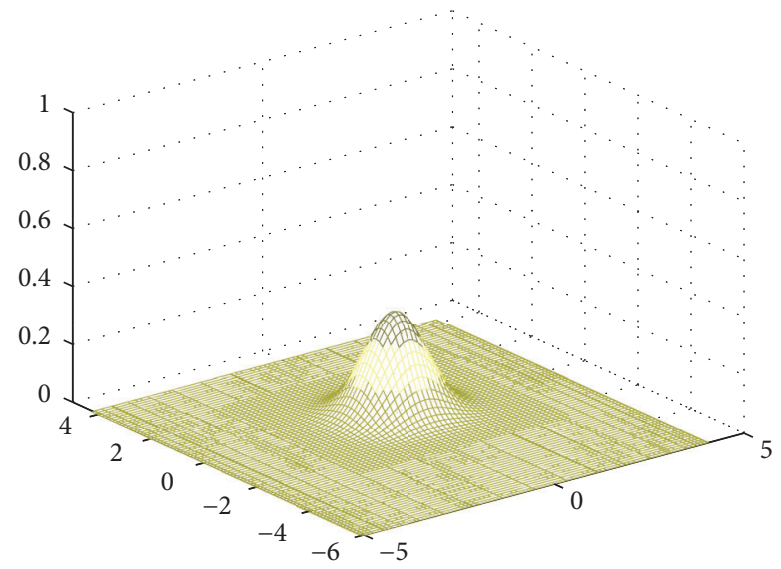

(b) $\omega=0$

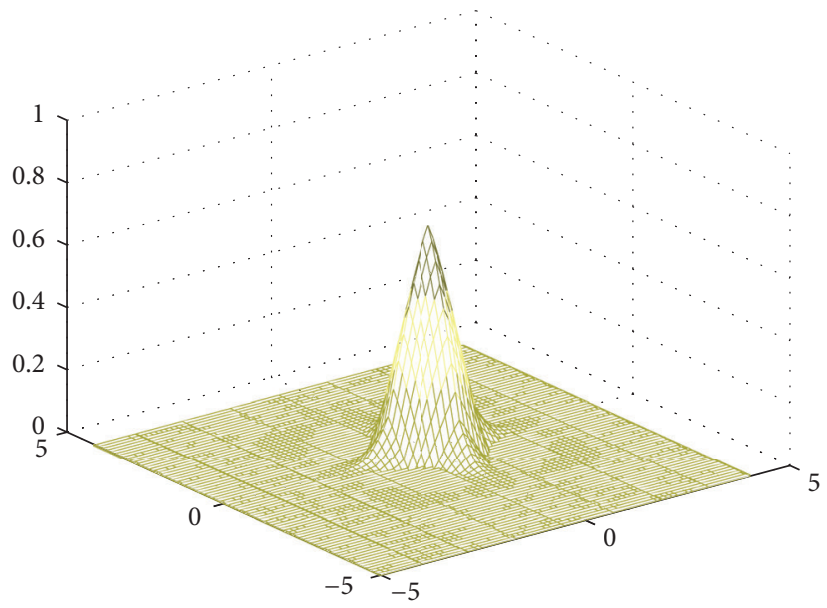

(c) $\omega=1 / 3$

FIGURE 6: Basic limit functions of subdivision scheme $S_{b_{1}}$ for three values of shape parameter $\omega$.

Proof. It is clear that

$$
\begin{aligned}
b_{1}(1,1) & =4, \\
b_{1}(1,-1) & =0, \\
b_{1}(-1,1) & =0, \\
b_{1}(-1,-1) & =0 .
\end{aligned}
$$

Let $n_{1}, n_{2} \in \mathbb{N}_{0}=\mathbb{N} \cup\{0\}$ and $\omega \in \Omega_{1}^{\prime}$, so

$$
\begin{gathered}
\left.D^{\left(n_{1}, n_{2}\right)} b_{1}\left(z_{1}, z_{2}\right)\right|_{z_{1}=1, z_{2}=-1}=0, \\
\left.D^{\left(n_{1}, n_{2}\right)} b_{1}\left(z_{1}, z_{2}\right)\right|_{z_{1}=-1, z_{2}=1}=0, \\
\left.D^{\left(n_{1}, n_{2}\right)} b_{1}\left(z_{1}, z_{2}\right)\right|_{z_{1}=-1, z_{2}=-1}=0,
\end{gathered}
$$

where $n_{1}+n_{2} \leq 1$. Therefore $S_{b_{1}}$ generates linear polynomials for $\omega \in \Omega_{1}^{\prime}$.
Again let $\omega=0$; then

$$
\begin{gathered}
\left.D^{\left(n_{1}, n_{2}\right)} b_{1}\left(z_{1}, z_{2}\right)\right|_{z_{1}=1, z_{2}=-1}=0, \\
\left.D^{\left(n_{1}, n_{2}\right)} b_{1}\left(z_{1}, z_{2}\right)\right|_{z_{1}=-1, z_{2}=1}=0, \\
\left.D^{\left(n_{1}, n_{2}\right)} b_{1}\left(z_{1}, z_{2}\right)\right|_{z_{1}=-1, z_{2}=-1}=0,
\end{gathered}
$$

where $n_{1}+n_{2} \leq 3$. Therefore $S_{b_{1}}$ generates cubic polynomials for $\omega=0$.

Theorem 10. The subdivision scheme $S_{b_{2}}$ generates

(i) quadratic polynomials for $\omega \in \Omega_{2}^{\prime}$,

(ii) quartic polynomials for $\omega=0$.

Proof. Proof is same as Theorem 9.

3.4. Reproduction Degree. Now we evaluate the polynomial reproduction of proposed family of schemes by using the methods given in [1] (Corollaries 2.7 and 2.3). 


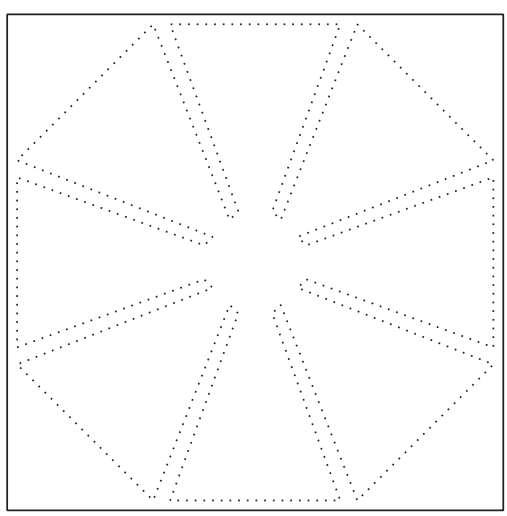

(a) Initial polygon

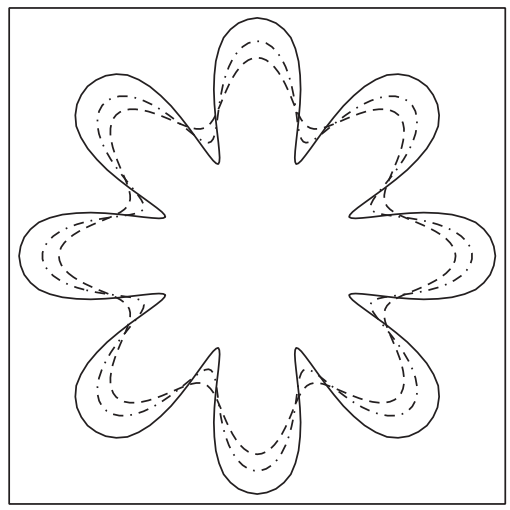

(c) $\omega=-1 / 20$

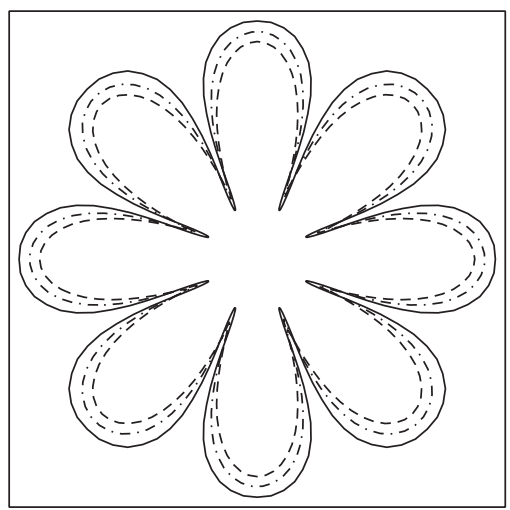

(e) $\omega=1 / 20$

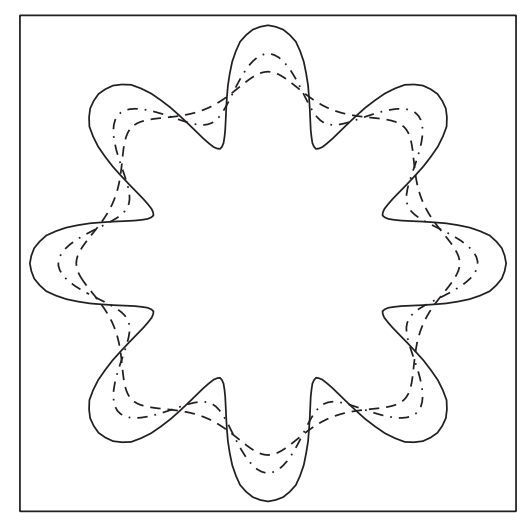

(b) $\omega=-1 / 10$

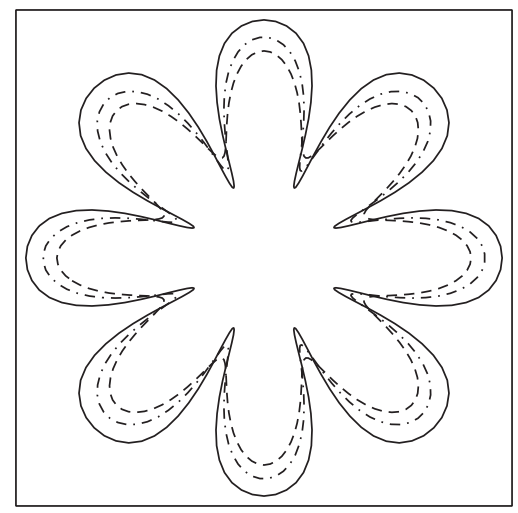

(d) $\omega=0$

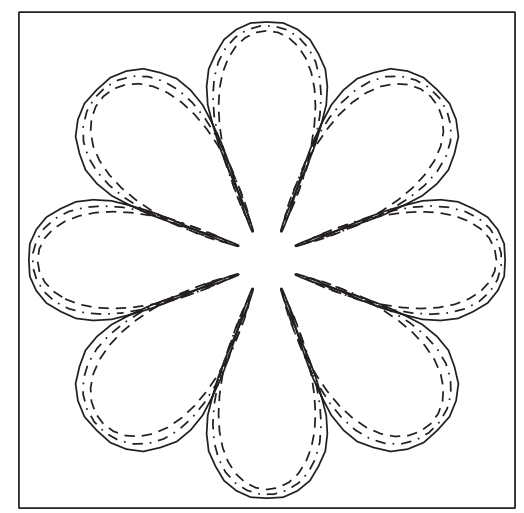

(f) $\omega=1 / 10$

Figure 7: In (b)-(f), solid lines, dashed dotted lines, and dash lines show the curves obtained by subdivision schemes $S_{a_{1}}$, $S_{a_{2}}$, and $S_{a_{3}}$, respectively.

Theorem 11. The subdivision schemes $S_{a_{l}}$ reproduce polynomials as follows:

(i) If $\omega \in \Omega_{l}, S_{a_{l}}$ reproduces linear polynomials.

(ii) If $\omega=23 / 216$ and $l=2, S_{a_{l}}$ reproduces quadratic polynomials.

(iii) If $\omega=(9+7 l) /(48+84 l)$ and $l>2, S_{a_{l}}$ reproduces cubic polynomials.
Proof. For $p \in \mathbb{N}$, the parametric value $\tau$ is given below:

$$
\tau= \begin{cases}0 & \text { if } l=2 p-1, \\ \frac{1}{2} & \text { if } l=2 p\end{cases}
$$

For $\omega \in \Omega_{l}$, we have $\left.D^{(1)} a_{l}(z)\right|_{z=1}=2 \tau$. Combining this condition with Theorem 8 , we get the required result. 


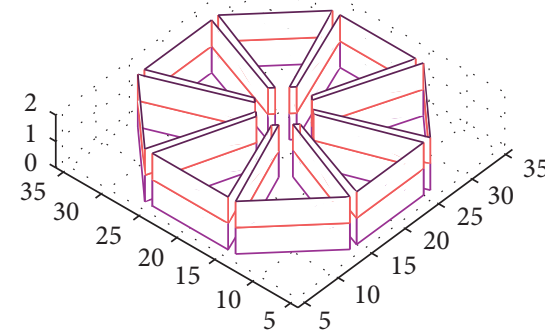

(a) Initial mesh

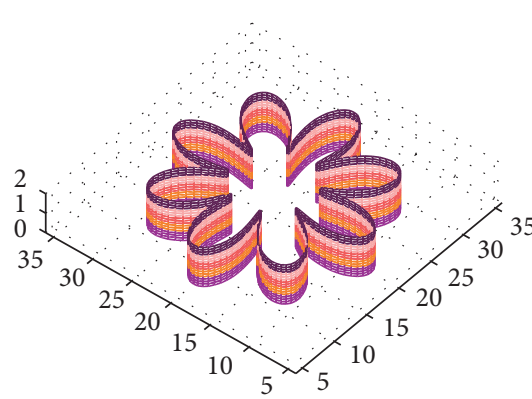

(c) $\omega=-1 / 10$

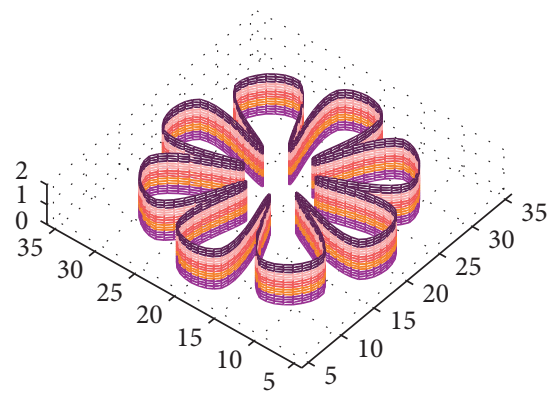

(e) $\omega=1 / 10$

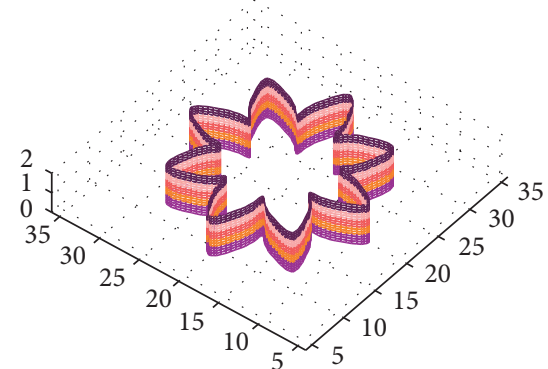

(b) $\omega=-1 / 3$

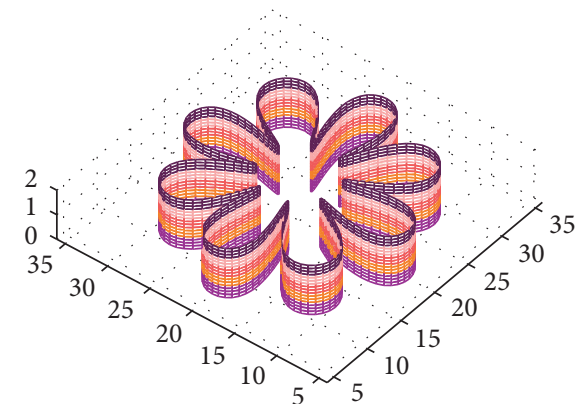

(d) $\omega=0$

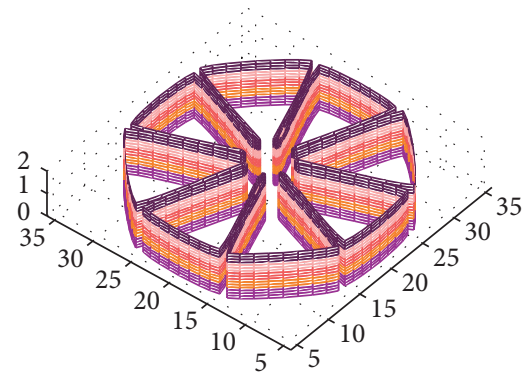

(f) $\omega=1 / 3$

FIGURE 8: (b)-(f) are limit surfaces obtained by applying one time our refining and one time smoothing operators for different values of shape parameter $\omega$ after 3 iterations.

Similarly for $\omega=23 / 216$ and $l=2, S_{a_{l}}$ reduces to the subdivision scheme with mask

$$
\begin{aligned}
a_{2} & =\left[-\frac{23}{10368},-\frac{53}{2592},\right. \\
& -\frac{137}{2592}, \frac{281}{10368}, \frac{1807}{5184}, \frac{907}{1296}, \frac{907}{1296}, \frac{1807}{5184}, \frac{281}{10368}, \\
& \left.-\frac{137}{2592},-\frac{53}{2592},-\frac{23}{10368}\right] .
\end{aligned}
$$

Since $\left.D^{(2)} a_{2}(z)\right|_{z=1}=-36 \omega+10 / 3$ then it is equal to $2 \tau(\tau-1)$ for $\omega=23 / 216 \in \Omega_{2}$. Now by combining this condition with Theorem 8 , we get the required result.

Again for $\omega=(9+7 l) /(48+84 l) \in \Omega_{l}$ and $l>2$, we have $\left.D^{(2)} a_{l}(z)\right|_{z=1}=2 \tau(\tau-1)$ and $\left.D^{(3)} a_{l}(z)\right|_{z=1}=2 \tau(\tau-1)(\tau-2)$. Once more by combining this condition with Theorem 8 , we get the result.
Theorem 12. The subdivision scheme $S_{b_{1}}$ reproduces linear polynomials for $\omega \in \Omega_{1}^{\prime}$.

Proof. Since

$$
\begin{aligned}
& \tau_{1}=\left.\frac{1}{4} D^{(1,0)} b_{1}\left(z_{1}, z_{2}\right)\right|_{z_{1}=1, z_{2}=1}=0 \\
& \tau_{2}=\left.\frac{1}{4} D^{(0,1)} b_{1}\left(z_{1}, z_{2}\right)\right|_{z_{1}=1, z_{2}=1}=0
\end{aligned}
$$

then

$$
\begin{aligned}
& 4 \tau_{1}=\left.D^{(1,0)} b_{1}\left(z_{1}, z_{2}\right)\right|_{z_{1}=1, z_{2}=1}=0 \\
& 4 \tau_{2}=\left.D^{(0,1)} b_{1}\left(z_{1}, z_{2}\right)\right|_{z_{1}=1, z_{2}=1}=0
\end{aligned}
$$

Now by combing these results with Theorem 9, the required result has been proved. 


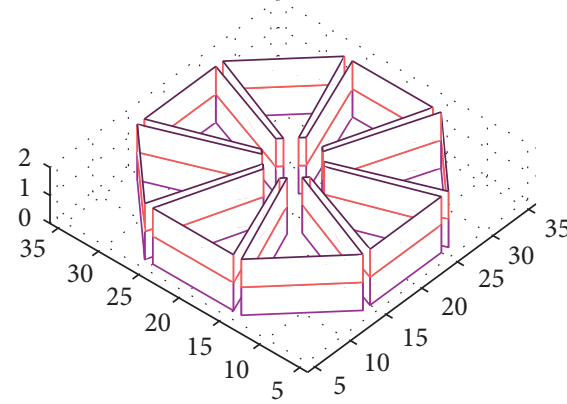

(a) Initial mesh

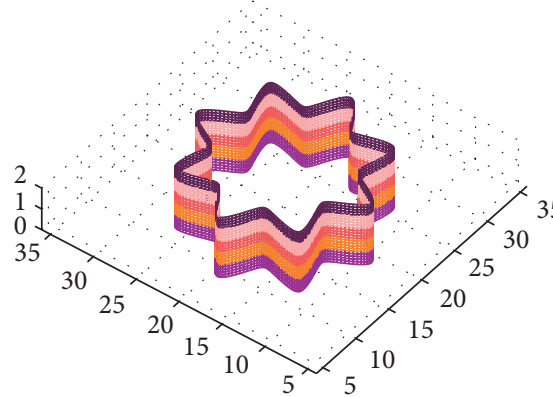

(c) $\omega=-1 / 3$

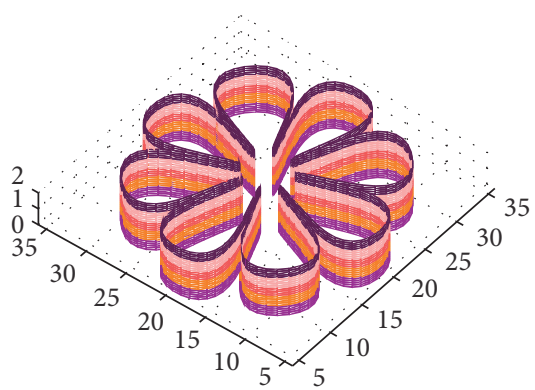

(e) $\omega=1 / 3$

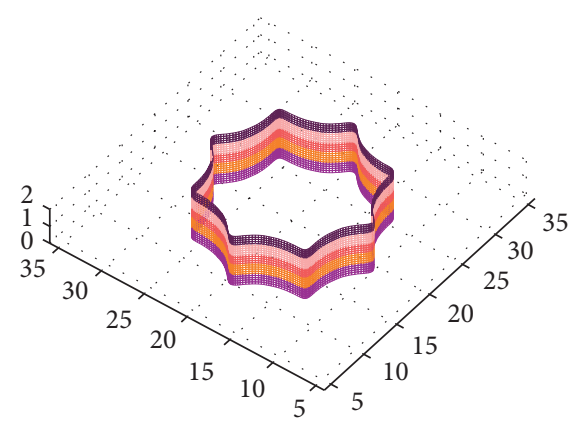

(b) $\omega=-1 / 2$

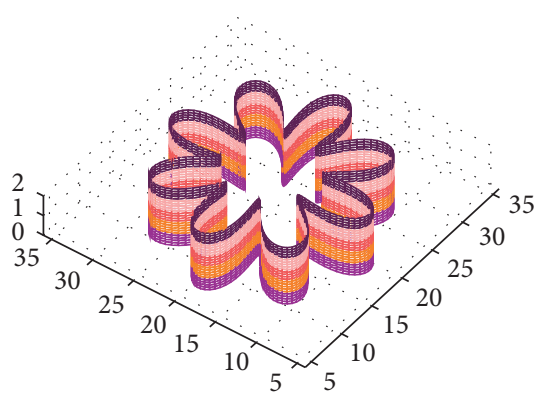

(d) $\omega=0$

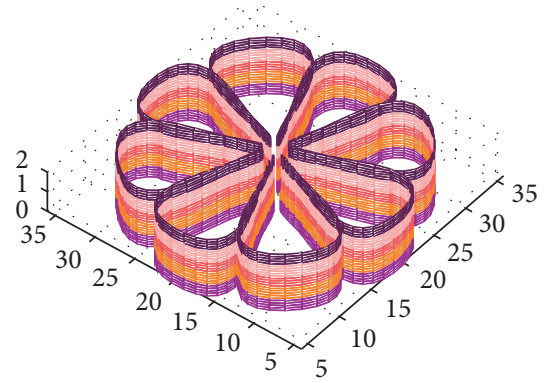

(f) $\omega=1 / 2$

FIGURE 9: (b)-(f) are limit surfaces obtained by applying one time our refining and two times our smoothing operators for different values of shape parameter $\omega$ after 3 iterations.

Theorem 13. The subdivision scheme $S_{b_{2}}$ reproduces linear polynomials for $\omega \in \Omega_{2}^{\prime}$ and quadratic polynomials for $\omega=$ $23 / 48$.

Proof. Since

$$
\begin{aligned}
& \tau_{1}=\left.\frac{1}{4} D^{(1,0)} b_{2}\left(z_{1}, z_{2}\right)\right|_{z_{1}=1, z_{2}=1}=\frac{1}{2}, \\
& \tau_{2}=\left.\frac{1}{4} D^{(0,1)} b_{2}\left(z_{1}, z_{2}\right)\right|_{z_{1}=1, z_{2}=1}=\frac{1}{2}
\end{aligned}
$$

then

$$
\begin{aligned}
& 4 \tau_{1}=\left.D^{(1,0)} b_{2}\left(z_{1}, z_{2}\right)\right|_{z_{1}=1, z_{2}=1}=2, \\
& 4 \tau_{2}=\left.D^{(0,1)} b_{2}\left(z_{1}, z_{2}\right)\right|_{z_{1}=1, z_{2}=1}=2 .
\end{aligned}
$$

Also, for $\omega=23 / 48$, we have

$$
\begin{aligned}
4 \tau_{1} \tau_{2} & =\left.D^{(1,1)} b_{2}\left(z_{1}, z_{2}\right)\right|_{z_{1}=1, z_{2}=1}=1, \\
4 \tau_{1}\left(\tau_{1}-1\right) & =\left.D^{(2,0)} b_{2}\left(z_{1}, z_{2}\right)\right|_{z_{1}=1, z_{2}=1}=-1, \\
4 \tau_{2}\left(\tau_{2}-1\right) & =\left.D^{(0,2)} b_{2}\left(z_{1}, z_{2}\right)\right|_{z_{1}=1, z_{2}=1}=-1 .
\end{aligned}
$$

Now by combing these results with Theorem 10 , the required result has been proved.

Remark 14. If we take $\omega=0$, the symbols (16) and (22) reduce to the symbol of "univariate family of subdivision schemes" and "bivariate family of subdivision schemes." These families produce $C^{l+2}$ limiting curves and surfaces, respectively. Also these families generate polynomials up to degree $l+2$, while polynomial reproduction of these families is linear. 


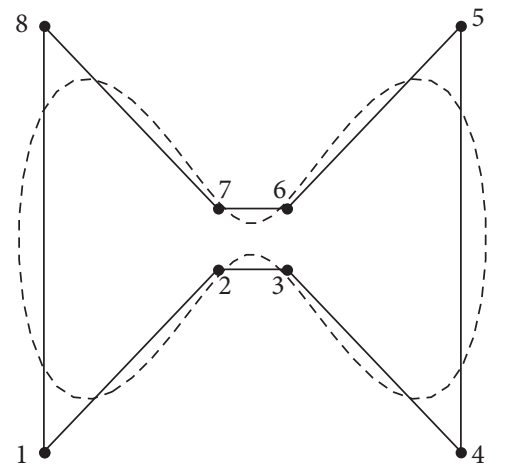

(a) Proposed uniform scheme $S_{a_{2}}, \omega=1 / 8$

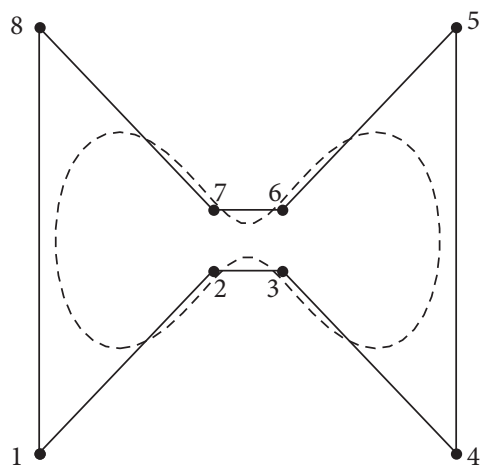

(c) Nonuniform proposed scheme $S_{a_{2}}$ $\omega_{i}=[1 / 32,1 / 8,1 / 8,1 / 32,1 / 32,1 / 8,1 / 8$, $1 / 32]$

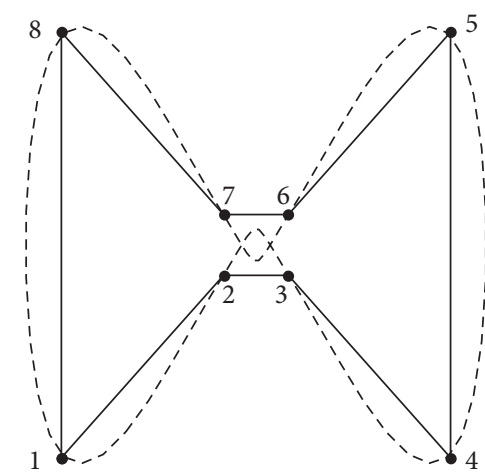

(b) Uniform Romani's scheme $S_{a_{2, \omega}}, \omega=$ $1 / 8$

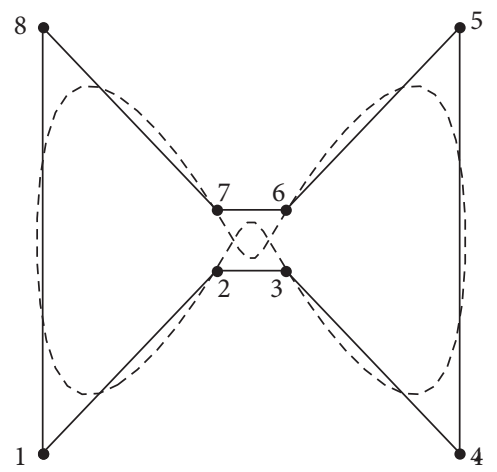

(d) Nonuniform Romani's scheme $S_{a_{2, \omega_{i}}}$, $\omega_{i}=[1 / 32,1 / 8,1 / 8,1 / 32,1 / 32,1 / 8,1 / 8$, $1 / 32]$

FIGURE 10: Solid lines represent initial polygons while dash lines represent limit curves obtained by subdivision schemes proposed ((a) and (c)) by us and ((b) and (d)) by Romani.

\section{Applications and Comparisons}

In this section, we have shown the capacity of parameter $\omega$ to control shapes in different ways. The optical presentation of our proposed families of univariate and bivariate (nontensor product for quadrilateral meshes) subdivision schemes also has been shown. In Figure 7, we have shown limit curves that are obtained by our proposed family of univariate subdivision schemes at five values of shape parameter. In Figures 8 and 9, we have also shown limit surfaces. These surfaces are obtained by $S_{b_{1}}$ (i.e., scheme with one time smoothing operator) and $S_{b_{2}}$ (i.e., scheme with two times smoothing operator) on quadrilateral meshes, respectively. From these figures, we observe that shapes of limit curves/surfaces are affected by shape parameter and number of applied smoothing stages both.

Tables 1 and 2 show comparison between our univariate and bivariate families with Romani's [8] univariate and bivariate families, respectively. From these tables it is clear that our schemes give better smoothness than Romani's schemes for $\omega=0$; however in this case the support size is larger than Romani's schemes and the reproduction degree is only one.

The geometrical construction of our refining operator allows us to extend our subdivision schemes to the nonuniform setting according to the geometry of the initial polygon/mesh by defining local parameter $\omega_{i}$. Sometimes choosing a variable shape parameter $\omega_{i}$ for each edge of the initial polygon/mesh gives better result than choosing a constant shape parameter $\omega$ for an entire initial polygon/mesh. Figures 10(a) and 10(b) demonstrate the visual effects of the proposed scheme $S_{a_{2}}$ and the scheme proposed by Romani $S_{a_{2, \omega}}$, respectively, for $\omega=1 / 8$. Similarly Figures 11(a) and $11(\mathrm{~b})$ display the visual performance of the proposed scheme $S_{b_{2}}$ and Romani's scheme $S_{b_{2, \omega}}$, respectively, for $\omega=1 / 8$, while Figures 10 (c) and 11(c) show the performance of nonuniform setting of the schemes $S_{a_{2}}$ and $S_{b_{2}}$, respectively. Also Figures $10(\mathrm{~d})$ and 11(d) represent the performance of nonuniform setting of Romani's schemes $S_{a_{2, \omega_{i}}}$ and $S_{b_{2, \omega_{i}}}$, respectively. These figures show that the limit curves/surfaces generated by nonuniform schemes are better than the limit curves/surfaces obtained from uniform schemes.

\section{Conclusion}

In this paper, we have presented univariate and bivariate families of approximating subdivision schemes depending on two parameters. These families are called the RefiningSmooth families where refining operator is the operator in 


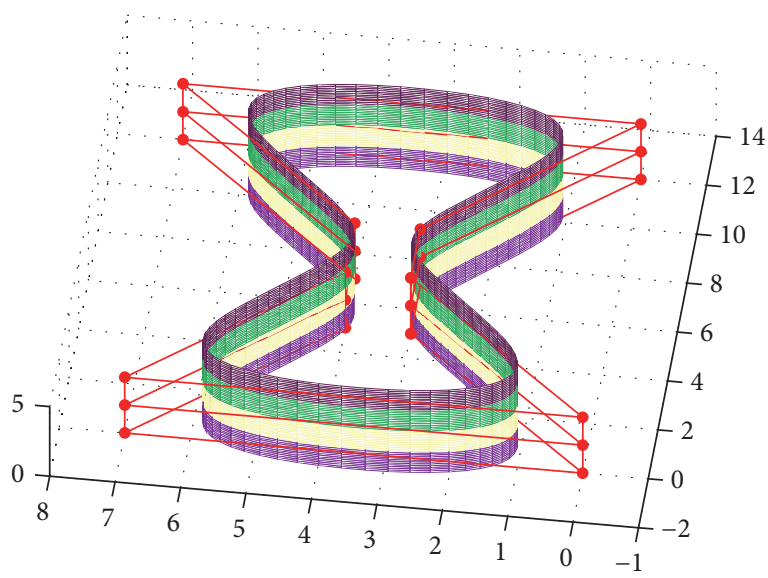

(a) Proposed uniform scheme $S_{b_{2}}, \omega=1 / 8$

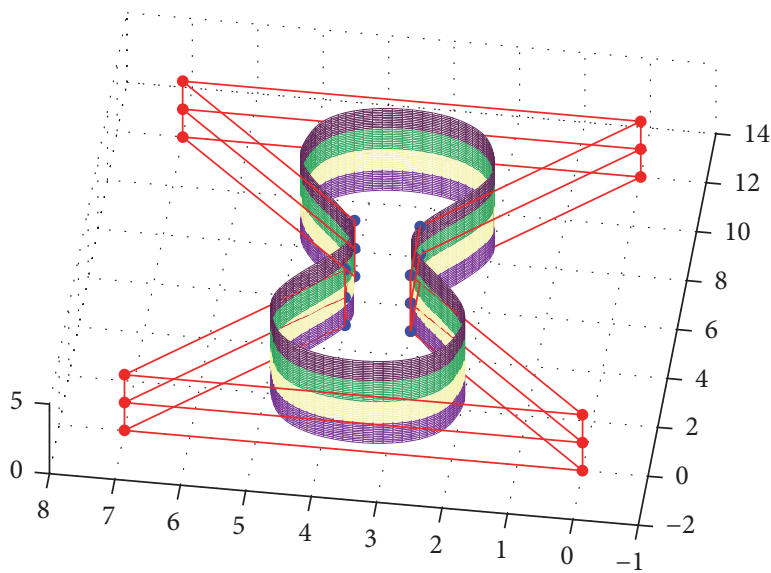

(c) Nonuniform proposed scheme $S_{b_{2}}, \omega_{i}=1 / 8$ at blue initial points and $\omega_{i}=0$ at red initial points

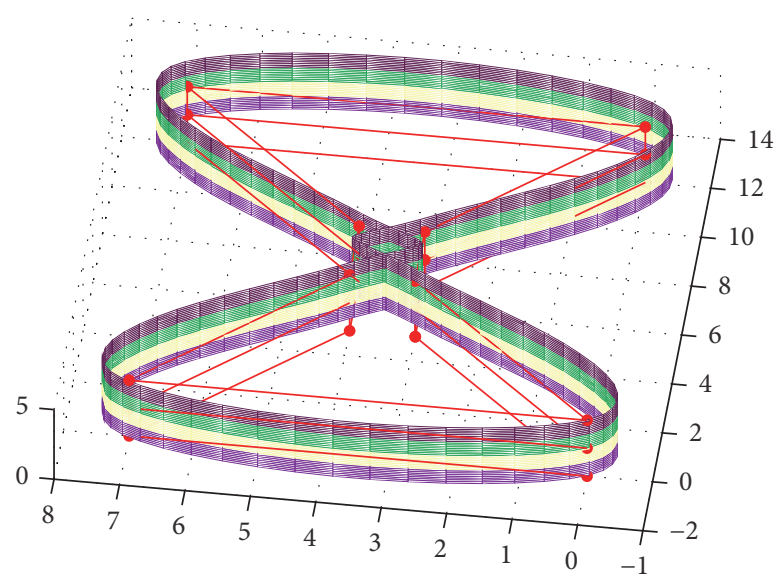

(b) Romani's uniform scheme $S_{b_{2, \omega}}, \omega=1 / 8$

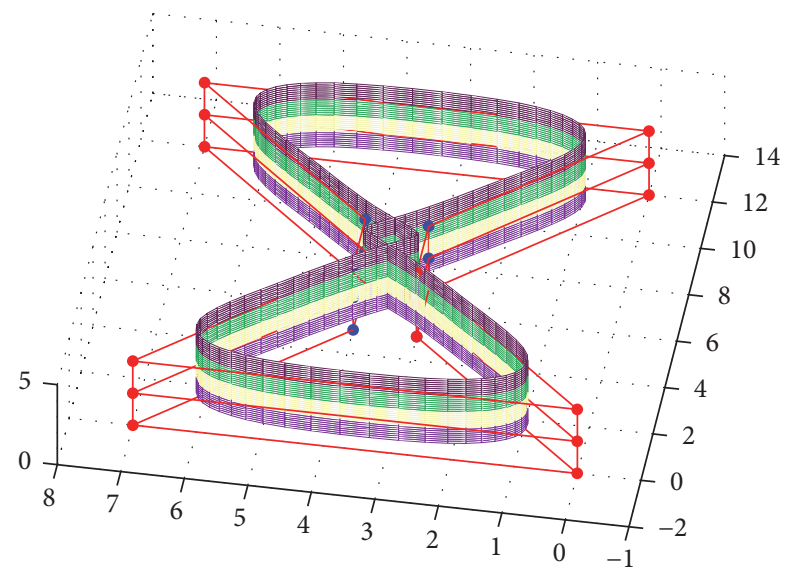

(d) Nonuniform Romani's scheme $S_{b_{2, \omega_{i}}}, \omega_{i}=1 / 8$ at blue initial points and $\omega_{i}=0$ at red initial points

FIGURE 11: Solid red lines represent initial mesh, while red and blue bullets show initial points.

TABLE 1: Comparison between our univariate family $S_{a_{l}}$ and Romani's univariate family $S_{a_{l, \omega}}$ via different properties. Let S, SS, RD, and GD be the integer smoothness, support size, reproduction degree, and generation degree, respectively.

\begin{tabular}{|c|c|c|c|c|}
\hline RS-schemes & $\mathrm{S}$ & SS & $\mathrm{RD}$ & GD \\
\hline$\overline{S_{a_{l}}}$ for $\omega \in \Omega_{l}$ & $C^{l}$ & $3 l+5$ & 1 & $l$ \\
\hline$S_{a_{l}}$ for $\omega=0 \in \Omega_{l}$ & $C^{l+2}$ & $3 l+3$ & 1 & $l+2$ \\
\hline$S_{a_{2}}$ for $\omega=23 / 216 \in \Omega_{2}$ & $C^{2}$ & 11 & 2 & 2 \\
\hline$S_{a_{l}}$ for $\omega=(9+7 l) /(48+84 l) \in \Omega_{l}$ and $l>2$ & $C^{l}$ & $3 l+5$ & 3 & $l$ \\
\hline$S_{a_{l, \omega}}$ for $\omega \in \Omega_{l}$ & $C^{l}$ & $l+5$ & 1 & $l$ \\
\hline$S_{a_{l, \omega}}$ for $\omega=1 / 16$ & $C^{l}$ & $l+5$ & 3 & $l+2$ \\
\hline
\end{tabular}

which each refining point $f_{i}^{k-1,1}$ moves around corresponding Chaikin point $C_{i}^{k-1,1}$ in curve case and each refining point $f_{i, j}^{k-1,1}$ moves around corresponding Doo-Sabin point $C_{i, j}^{k-1,1}$ in surface case for different values of shape parameter $\omega \epsilon$ $\mathbb{R}$ and smoothing parameter $l \in \mathbb{N}$, while each smoothing operator is the weighted average of the four adjacent points $f_{i}^{k-1, n}, i \in \mathbb{Z}$, in curve case and each smoothing operator is the weighted average of sixteen adjacent neighboring points $f_{i, j}^{k-1, n}, i, j \in \mathbb{Z}$, in surface case, where $n=1,2, \ldots, l$.

We also have analyzed some basic properties of our proposed families. Our families of schemes give cubic polynomial reproduction for specific values of shape parameter $\omega$. Polynomial generation and continuity of our schemes are $l$ for specific range of parameter $\omega$, while for $\omega=0$ they are $l+2$. However for $\omega=0$, our schemes reproduce only linear 
TABLE 2: Comparison between our bivariate family $S_{b}$ and Romani's bivariate family $S_{b_{l, \omega}}$ via different properties. Let $S, S S, R D$, and GD be the integer smoothness, support size, reproduction degree, and generation degree, respectively.

\begin{tabular}{lcccc}
\hline RS-schemes with $l \leq 2$ & S & SS & RD & GD \\
\hline$S_{b_{l}}$ for $\omega \in \Omega_{l}^{\prime}$ & $C^{l}$ & $(3 l+3) \times(3 l+3)$ & 1 & $l$ \\
$S_{b_{l}}$ for $\omega=0 \in \Omega_{l}^{\prime}$ & $C^{l+2}$ & $(3 l+3) \times(3 l+3)$ & 1 & $l+2$ \\
$S_{b_{2}}$ for $\omega=23 / 48 \in \Omega_{2}^{\prime}$ & $C^{1}$ & $9 \times 9$ & 2 & 2 \\
$S_{b_{l, \omega}}$ for $\omega \in \Omega_{l}^{\prime}$ & $C^{l}$ & $(l+5) \times(l+5)$ & 1 & $l$ \\
$S_{b_{l, \omega}}$ for $\omega=1 / 16$ & $C^{l}$ & $(l+5) \times(l+5)$ & 3 & $l+2$ \\
\hline
\end{tabular}

polynomials. Nonuniform setting of the proposed family members gives better performance than that of uniform setting of the schemes.

\section{Competing Interests}

The authors declare that they have no competing interests.

\section{Acknowledgments}

This work is supported by NRPU of HEC Pakistan (P. no. 3183).

\section{References}

[1] M. Charina and C. Conti, "Polynomial reproduction of multivariate scalar subdivision schemes," Journal of Computational and Applied Mathematics, vol. 240, pp. 51-61, 2013.

[2] N. Dyn, "Interpolatory subdivision schemes and analysis of convergence and smoothness by the formalism of Laurent polynomials," in Tutorials on Multiresolution in Geometric Modeling, A. Iske, E. Quak, and M. S. Floater, Eds., pp. 51-68, Springer, Berlin, Germany, 2002.

[3] N. Dyn and D. Levin, "Subdivision schemes in geometric modelling," Acta Numerica, vol. 11, pp. 73-144, 2002.

[4] E. Catmull and J. Clark, "Recursively generated B-spline surfaces on arbitrary topological meshes," Computer-Aided Design, vol. 10, no. 6, pp. 350-355, 1978.

[5] D. Doo and M. Sabin, "Behaviour of recursive division surfaces near extraordinary points," Computer-Aided Design, vol. 10, no. 6, pp. 356-360, 1978.

[6] M.-E. Fang, W. Ma, and G. Wang, "A generalized surface subdivision scheme of arbitrary order with a tension parameter," Computer-Aided Design, vol. 49, pp. 8-17, 2014.

[7] J. M. Lane and R. F. Riesenfeld, "A theoretical development for the computer generation and display of piecewise polynomial surfaces," IEEE Transactions on Pattern Analysis and Machine Intelligence, vol. 2, no. 1, pp. 35-46, 1980.

[8] L. Romani, "A Chaikin-based variant of Lane-Riesenfeld algorithm and its non-tensor product extension," Computer Aided Geometric Design, vol. 32, pp. 22-49, 2015.

[9] J. Stam, "On subdivision schemes generalizing uniform B-spline surfaces of arbitrary degree," Computer Aided Geometric Design, vol. 18, no. 5, pp. 383-396, 2001.

[10] D. Zorin and P. Schröder, "A unified framework for primal/dual quadrilateral subdivision schemes," Computer Aided Geometric Design, vol. 18, no. 5, pp. 429-454, 2001.
[11] P. Ashraf, G. Mustafa, and J. Deng, "A six-point variant on the Lane-Riesenfeld algorithm," Journal of Applied Mathematics, vol. 2014, Article ID 628285, 7 pages, 2014.

[12] T. J. Cashman, K. Hormann, and U. Reif, "Generalized LaneRiesenfeld algorithms," Computer Aided Geometric Design, vol. 30, no. 4, pp. 398-409, 2013.

[13] G. Mustafa, P. Ashraf, and M. Aslam, "Binary univariate dual and primal subdivision schemes," SEMA Journal, vol. 65, no. 1, pp. 23-35, 2014.

[14] G. Deslauriers and S. Dubuc, "Symmetric iterative interpolation processes," Constructive Approximation, vol. 5, no. 1, pp. 49-68, 1989.

[15] N. Dyn, D. Levin, and J. A. Gregory, "A 4 -point interpolatory subdivision scheme for curve design," Computer Aided Geometric Design, vol. 4, no. 4, pp. 257-268, 1987.

[16] K. Hormann and M. A. Sabin, "A family of subdivision schemes with cubic precision," Computer Aided Geometric Design, vol. 25, no. 1, pp. 41-52, 2008.

[17] G. M. Chaikin, "An algorithm for high-speed curve generation," Computer Graphics and Image Processing, vol. 3, no. 4, pp. 346$349,1974$.

[18] I. P. Ivrissimtzis, M. A. Sabin, and N. A. Dodgson, "On the support of recursive subdivision," ACM Transactions on Graphics, vol. 23, no. 4, pp. 1043-1060, 2004. 


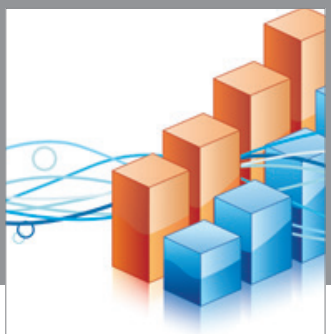

Advances in

Operations Research

vatem alat4

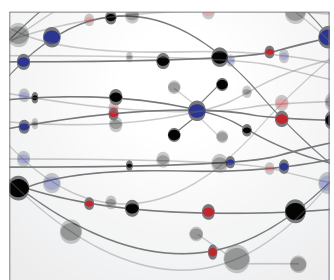

\section{The Scientific} World Journal
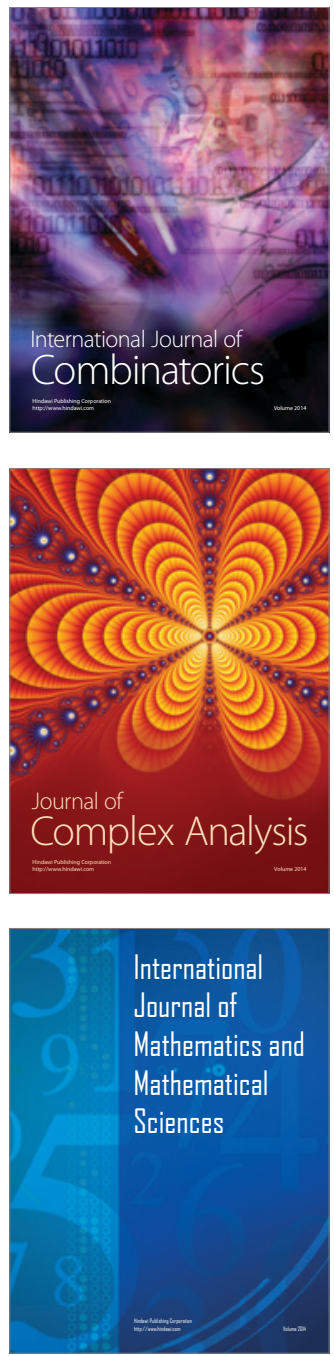
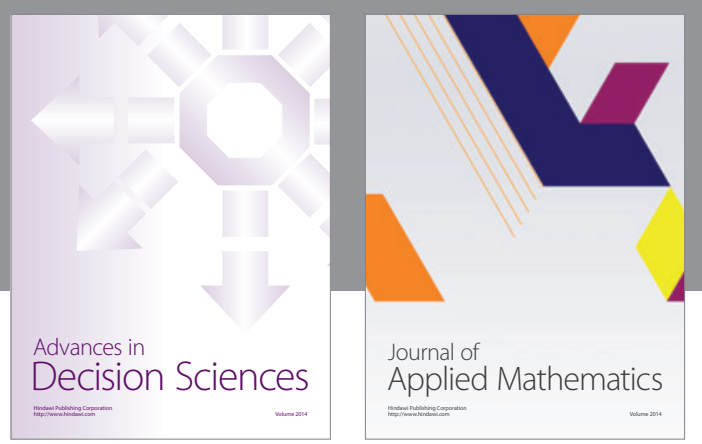

Algebra

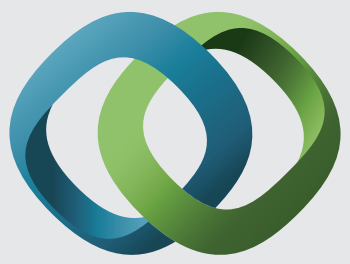

\section{Hindawi}

Submit your manuscripts at

http://www.hindawi.com
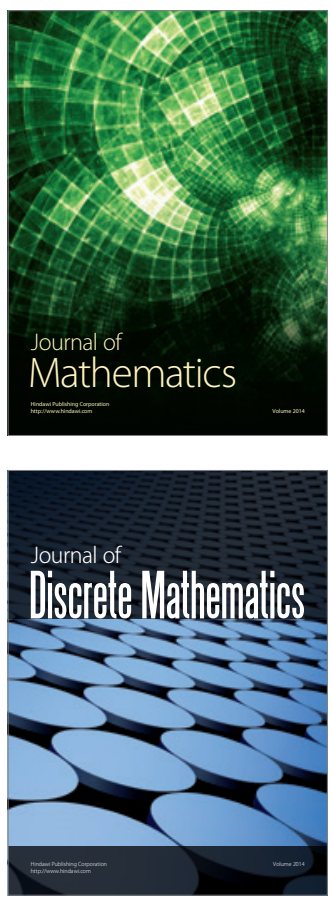

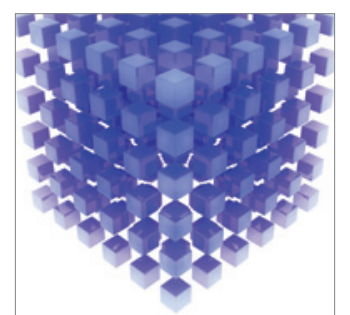

Mathematical Problems in Engineering
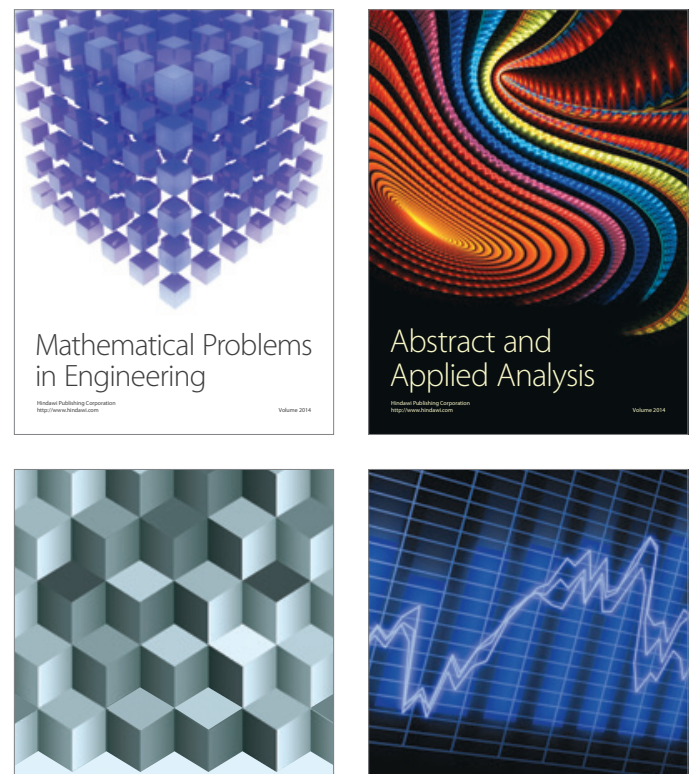

Journal of

Function Spaces

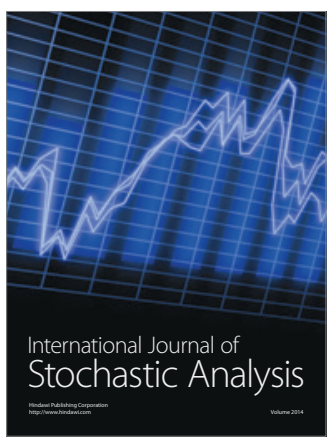

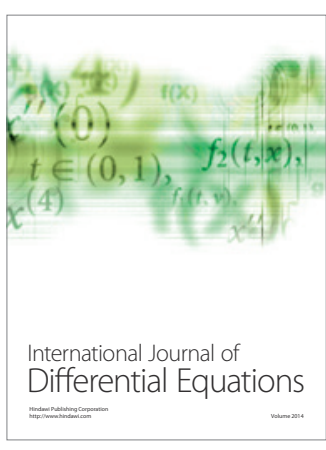
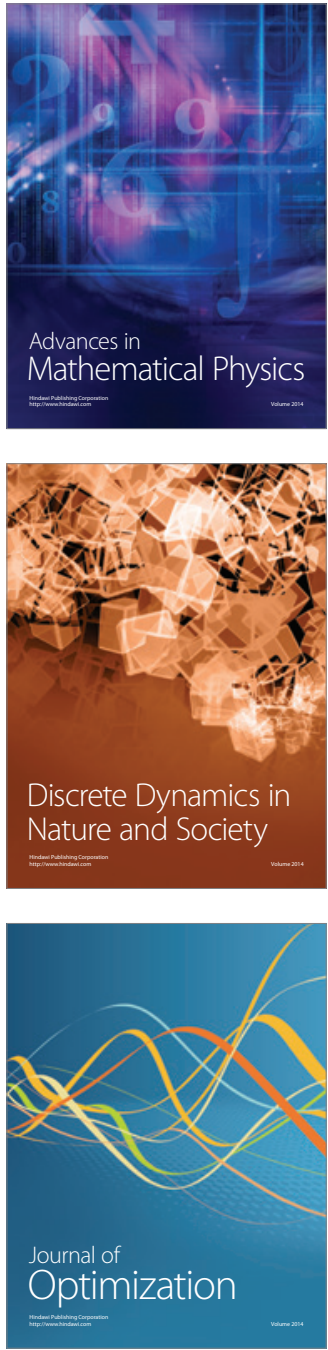